\title{
Preliminary Results of Shake Table Tests of a Typical Museum Display Case Containing an Art Object
}

\author{
Andrea Prota $\triangle{ }^{1},{ }^{1}$ Martino Zito $\mathbb{D},{ }^{1}$ Danilo D'Angela $\mathbb{D}^{1},{ }^{1}$ Giuseppe Toscano, ${ }^{1}$ \\ Carla Ceraldi $\left(\mathbb{D},{ }^{1}\right.$ Antimo Fiorillo, ${ }^{1}$ and Gennaro Magliulo $\mathbb{D}^{1,2}$ \\ ${ }^{1}$ Department of Structures for Engineering and Architecture, University of Naples Federico II, Naples 80125, Italy \\ ${ }^{2}$ Construction Technologies Institute, National Research Council, Naples 80125, Italy \\ Correspondence should be addressed to Gennaro Magliulo; gmagliul@unina.it
}

Received 24 September 2021; Accepted 10 January 2022; Published 27 January 2022

Academic Editor: Daniele Perrone

Copyright (C) 2022 Andrea Prota et al. This is an open access article distributed under the Creative Commons Attribution License, which permits unrestricted use, distribution, and reproduction in any medium, provided the original work is properly cited.

\begin{abstract}
The seismic response of freestanding elements is typically extremely critical, and this is associated with a high seismic risk in the case of museum objects and artefacts. This paper reports the preliminary results of an experimental testing campaign aimed at assessing the dynamic properties and the seismic performance of museum objects and artefacts. Shake table tests of a typical museum display case containing a representative art object (vase) are performed. Both dynamic properties and seismic behavior of the specimens were assessed. Tentative damage assessment correlations are developed. The study sheds light on the critical behavior of the tested specimens, stressing the need for further studies towards a more comprehensive assessment of freestanding museum objects and artefacts.
\end{abstract}

\section{Introduction}

The seismic response of engineering systems governed by rigid motion (e.g., freestanding or unanchored elements) is typically extremely critical [1-4]. In many cases, rigiddominated systems are nonstructural elements [5-8]. The seismic risk associated with these systems can be high, especially if they are housed within critical facilities (e.g., hospital or laboratory equipment $[9,10]$ or nuclear facilities $[11,12])$ or have historical/cultural significance (e.g., art objects) [13-15]. In the light of that, several studies recently provided guidance for mitigating the seismic vulnerability of freestanding valuable and museum systems and objects [16-18]. However, current arrangements for those systems housed in critical facilities and museums typically do not include protection systems, except for few peculiar cases.

Freestanding systems typically exhibit significant rocking-sliding motion under relatively low seismic intensity excitation [19-21]. Several studies assessed the seismic response of critical freestanding (or unanchored) elements through experimental testing $[9,22-25]$ and numerical analysis [12, 20, 26-29]. Dar et al. [12] assessed the reliability of simplified method provided by ASCE 43-05 for the assessment of freestanding elements. They found that this method provides unreliable estimations, recommending the use of more refined analysis methodologies (nonlinear dynamic analyses). Fragiadakis and Diamantopoulos [28] developed a simplified approach to assess the fragility of freestanding building contents and provided promising quantitative results for risk estimates regarding a case study application (four-story reinforced concrete building). Huang et al. [30] performed free-rocking tests on freestanding elements accounting for variation in center of gravity location. Particular focus was on rocking response and dynamic properties and on the influence of the center of gravity variation on these parameters. The study highlighted potential discrepancies between analytical estimations and experimental response, providing an accurate equivalent rectangular rigid block model.

Despite the copious literature addressing the seismic response of freestanding elements, a small number of studies focused on museum objects and artefacts. Berto et al. [31] investigated the seismic behavior of six Michelangelo's sculptures by means of rigid block and finite element 
analysis. They highlighted the weakness of the investigated statues regarding their seismic performance and provided technical insights about the mitigation of the seismic risk associated with art objects. Sorace and Terenzi [17] carried out finite element analysis of a marble statue housed in a castle in Italy through an incremental procedure, assessing both dynamic behavior and stress distribution. The case study was representative of a wide range of scenarios. They developed assessment criteria and suggested possible isolation solutions for an effective seismic protection of art objects. Wittich and Hutchinson [24] performed shake table tests on statue-pedestal unattached systems, varying geometry (including asymmetry), loading history, and friction coefficient. They stressed the complexity of the dynamic response of dual body unanchored systems, characterized by both significant sliding and rocking, multimodal interaction, and three-dimensional response.

Very few studies focused on freestanding display cases/ cabinets containing unanchored art objects or valuable components. This configuration is typically quite critical since both the container (e.g., display case) and the content (e.g., art object) may exhibit rigid-dominated response, associated with major motion and resulting in high seismic risk. Moreover, most of small and medium size objects and components are often contained in such display cases or cabinets, and this makes this configuration quite common in museums and critical facilities. Neurohr and McClure [32] performed shake table testing of high density fiberboard display cases with Plexiglas covers containing art objects. They assessed the influence of seismic input, floor height, surface friction, and mass of art objects on the vulnerability of the investigated specimens. Cosenza et al. [19] and Di Sarno et al. [9] performed shake table tests of freestanding hospital cabinets containing unanchored containers and phials. They investigated the influence of the contents on the dynamic properties and seismic performance of the cabinets and assessed the seismic fragility of both cabinets and contents.

Despite the recent research efforts towards a reliable seismic assessment and an effective protection of freestanding components and art objects, dynamic properties and seismic vulnerability of the most common configurations of museum systems and objects are still unknown. The present study represents a first step carried out to fill the abovementioned research gap. In particular, shake table tests of a steel-glass display case containing an unanchored art object (vase) are performed, and preliminary results are reported in this paper. The specimens represent a very common museum configuration, potentially associated with a high vulnerability. The specimens were provided by the National Archaeological Museum of Naples (MANN), Italy. The dynamic properties and seismic behavior of both display case and art object are assessed. A preliminary damage assessment is also performed in the light of the observations and the data analysis.

1.1. Display Case and Art Object. The tested display case is part of the exhibition equipment of the MANN (Figure 1). The dimensions of the assembled display case are

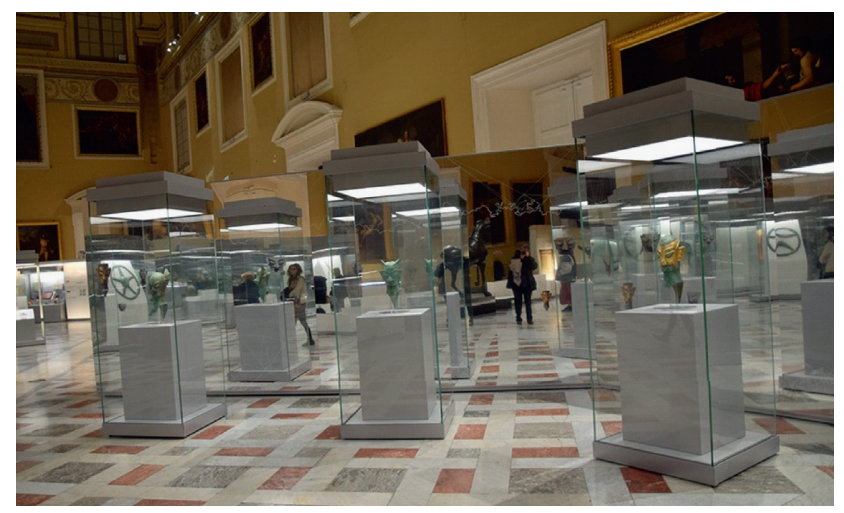

Figure 1: Arrangement of the tested display case within the $\mathrm{Na}$ tional Archaeological Museum of Naples (MANN), Italy.

$92 \times 92 \times 244.5 \mathrm{~cm}$. The display case is composed of several components that were assembled on the shake table by specialized workers. The display case assembled on the shake table is depicted in Figure 2(a). The steel base frame has dimensions of $92 \times 92 \times 12 \mathrm{~cm}$ (width $\times$ length $\times$ height $)$ and weight of $46.2 \mathrm{~kg}$ (Figure 2(b)). Four corner supports are screwed below the base frame, and each support is made of a steel threaded rod having a circular base pin, covered in plastic (Figure 2(c)); the supports have $1.5 \mathrm{~cm}$ height. A cover plate is simply supported by the internal part of the base frame. The plate consists of an internal wood plate covered by a steel shelter; the plate has dimensions of $84 \times 84 \times 1.8 \mathrm{~cm}$ (width $\times$ length $\times$ thickness) and weight of $16.3 \mathrm{~kg}$. The lateral surface of the case consists of three fixed panels and a front rotating door panel (Figure 2(a)). Both fixed panels and door panel are made of double-glass panels fixed to an inferior and a superior thin steel runner. Each glass panel has dimensions of $88.5 \times 234 \times 1.1 \mathrm{~cm}$ (width $\times$ height $\times$ thickness) and mass of about $55 \mathrm{~kg}$. The door rotates about two hinges, fastened to the top and base frame; the door has an inferior and superior locker, fixing the runners to the base and top frames (Figure 2(a)). Both top and bottom door runners have dimensions equal to $92 \times 2 \mathrm{~cm}$ (length $\times$ thickness) and mass of about $10.5 \mathrm{~kg}$. The four lateral panels are connected to a top frame, having mass of $58.8 \mathrm{~kg}$, that also incorporates the superior lighting system (Figure 2(d)); the top cover frame has height equal to $12 \mathrm{~cm}$, equal to the height of the base frame. The glass panels, including the opening glass panel, are fixed to the bottom and top elements by friction. In particular, both bottom and top elements (corresponding to the runners) have a bucket (with seals) that is made by two flanges connected by a screw and two bolts (Figure 2(e)); the glass panels are inserted within this bucket, and the applied torque (fastening the screw/bolts) provides the friction to fix the glass panels to those flanges, causing their fastening to base and top elements.

A pedestal is located within the case and is simply supported by the internal cover plate (Figure 2(a)). The pedestal is made of a welded steel frame and thin steel cover surfaces; the frame consists of welded square hollow thin sections with dimensions of $3 \times 3 \mathrm{~cm}$; the top and lateral pedestal steel covers are welded 


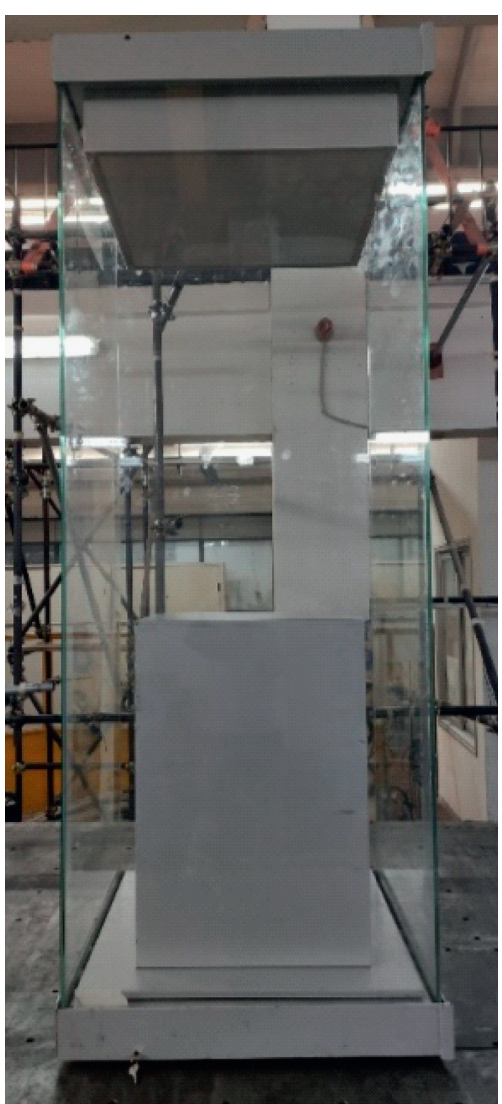

(a)

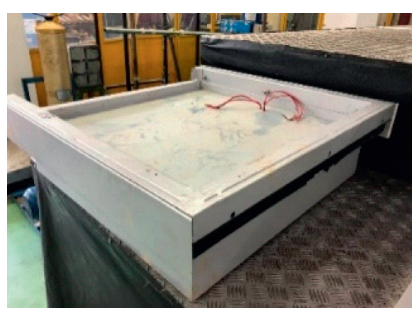

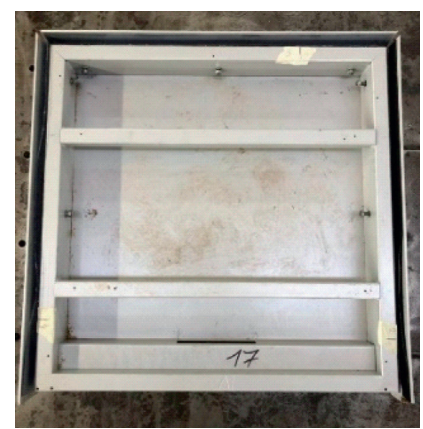

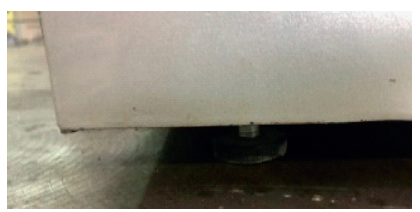

(b)

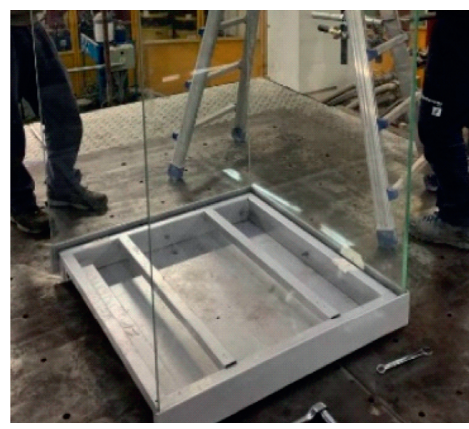

(d) (e) (c)

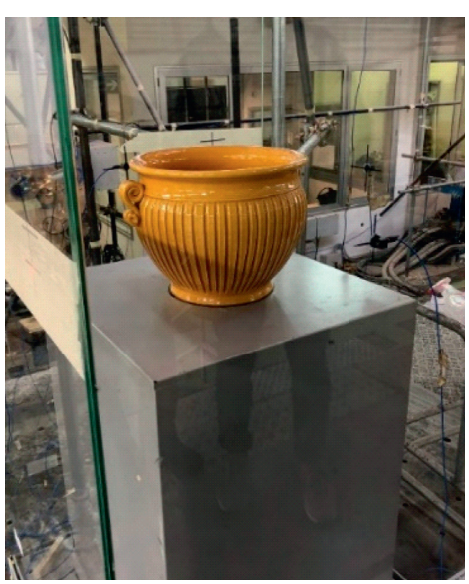

(f)

FIGURE 2: Details of the tested specimen: (a) assembled display case, (b) base frame, (c) detail of the base frame support, (d) upside down top frame, (e) detail of the glass to frame connections, and (f) detail of pedestal and art object (vase).

to the frame and have $2 \mathrm{~cm}$ width, whereas the base frame does not have a cover. The pedestal has dimensions of $60 \times 60 \times 88 \mathrm{~cm}$ (width $\times$ length $\times$ height $)$ and mass of $45.8 \mathrm{~kg}$. An art object was placed on the artefact support (Figure 2(e)). The object was a ceramic vase, having base diameter and maximum diameter plus handles equal to about 22 and $42 \mathrm{~cm}$, respectively; height equal to about $29 \mathrm{~cm}$; and mass equal to about $7 \mathrm{~kg}$.

The display case, including the art object, is an assembly of several parts. The base and top frames of display case are relatively rigid and are interconnected through the lateral glass panels (including the door). The glass panels are fastened to the base and top frame runners by means of friction bucket connections, which might allow a relatively reduced amount of free (relative) rotation. The components located inside the display case, i.e., basement, pedestal, and art object, are not fastened to each other (they are just freestanding), and the basement is not fastened to the display case frame (just freestanding). Therefore, basement, pedestal, and art object are expected to be governed by a rigid 
block response; in particular, the basement is expected to exhibit a sliding response due to the very reduced slenderness; the pedestal is expected to be mostly governed by sliding due to reduced friction (smooth contact surfaces); the art object is expected to exhibit sliding due to reduced slenderness and reduced friction. The whole display case system is expected to exhibit a mixed sliding-rocking response, given the relatively slender geometry (rocking) and the relatively reduced friction (sliding). The sliding response might be more significant than the rocking one. The display case is also expected to exhibit minor elastic vibrational response (especially glass vibrations out of their plane). The dynamics of the internal components might condition the overall response of the system, especially the rigid-dominated motion. This description is only meant to ease the interpretation of the experimental results and does not involve numerical modeling or analysis.

\section{Shake Table Testing}

2.1. Testing Set-Up and Instrumentation. The display case was simply supported by the shake table steel floor, and the door panel was facing north side. Shake table and tested specimens were monitored by accelerometers (Acc), displacement laser sensors (Las), and video cameras (Figure 3). Four triaxial accelerometers (Acc054, Acc658, Acc766, and Acc056) were installed at the center of the double-glass panels, on the external surface of the glass. Two triaxial accelerometers (Acc052 and Acc053) were installed at the center of the east and south sides of the base frame. On south and west sides, two triaxial accelerometers (Acc765 and Acc763) were positioned at the center of the top frame. A triaxial accelerometer (Acc050) was installed on the south side to measure the response of the bottom portion of the double-glass panel with respect to the two flanges. Two triaxial accelerometers (Acc762 and Acc818) were placed on the artefact support and base frame cover plate, respectively. The acceleration of the table was monitored by an internal accelerometer (AccT). Six displacement laser sensors were installed on the south side (LasL2, LasW3, and LasW2) and east side (LasL3, LasW6, and LasW5). Two sensors (LasW1 and LasW4) were placed on the south and east sides, respectively, in order to record the displacement of the shake table in both horizontal directions. A safety scaffolding structure was built around the display case to protect the instruments and prevent critical displacement/overturning. The structure was $25 \mathrm{~cm}$ distant from the display case.

2.2. Testing Input and Program. Shake table testing represents the most reliable method for seismic assessment of acceleration-sensitive elements such as freestanding systems $[1,24,33]$. Seismic assessment and qualification of acceleration-sensitive elements are typically performed through two types of testing: dynamic identification and seismic performance assessment [34, 35]. Dynamic identification and seismic performance tests were performed through mono- and bidirectional shake table testing, respectively. Low-amplitude random vibration signals (i.e., maximum acceleration not exceeding $0.1 \mathrm{~g}$ ) were used to perform the dynamic identification tests (i.e., random (RAN) tests) [9]. The seismic performance tests were carried out according to an incremental procedure, whereas the dynamic identification tests were performed prior to, during, and after the incremental tests. The shake table input related to the incremental tests (AC tests) was developed according to the ICC-ES AC156 protocol [35], which represents the international reference for seismic qualification and certification of acceleration-sensitive nonstructural components [1]. The procedure developed in [36] was used to generate/process the testing signals; this was already used in other experimental studies carried out by the authors (e.g., [9, 37-39]); further details are omitted as the procedure is well described in the abovementioned literature studies. It should be noted that AC156 protocol is not aimed at assessing freestanding elements but generic acceleration-sensitive elements, which are typically meant to be single-point attached/fastened to the structure. However, this protocol is typically used in literature and practice also for these components $[9,19,33,40-42]$ since no alternative reference protocols exist to assess and qualify freestanding elements. For more details regarding this issue, please refer to $[1,29]$.

The design spectral acceleration at short periods $S_{D S}$ was used as a reference intensity measure for scaling the intensity of the incremental tests. The acceleration time history and the response spectra associated with the defined AC156 input along both horizontal directions are depicted in Figures 4(a) and 4(b), respectively, considering $S_{D S}$ equal to $0.45 \mathrm{~g}$. In particular, test response spectra (TRS) and required response spectra (RRS) are depicted in Figure 4(b) considering sixths of octave as a unit reference along the ordinate axis. Please refer to [38] for further details regarding the development of the testing inputs.

Table 1 reports the testing program, including both dynamic identification random tests, i.e., RAN tests, and seismic performance AC156 tests, i.e., AC tests. The table maximum acceleration recorded along both horizontal directions, meant to be a peak floor acceleration (PFA), is also reported in Table 1.

2.3. Analysis and Assessment Methodology. The spectrum compatibility between TRS and RRS was assessed according to the procedure defined by AC156 protocol $[35,43]$. The dynamic identification of the specimens was carried out, and both natural frequencies and damping ratios of the specimens were assessed [44-46]. The transfer function method was used to estimate the natural frequency of the tested museum display case. In particular, the transfer function of the tested museum display case was obtained as the ratio of the Fourier transforms related to acceleration time histories recorded at the display case top and at the shake table.

The equivalent damping ratio was evaluated according to the half-power bandwidth method $[46,47]$, typically used to assess structures and components assumed to have linear viscous damping $[44,48]$. The damping ratio associated with the first mode of the display case was evaluated by data from each frequency response obtained by dynamic identification 

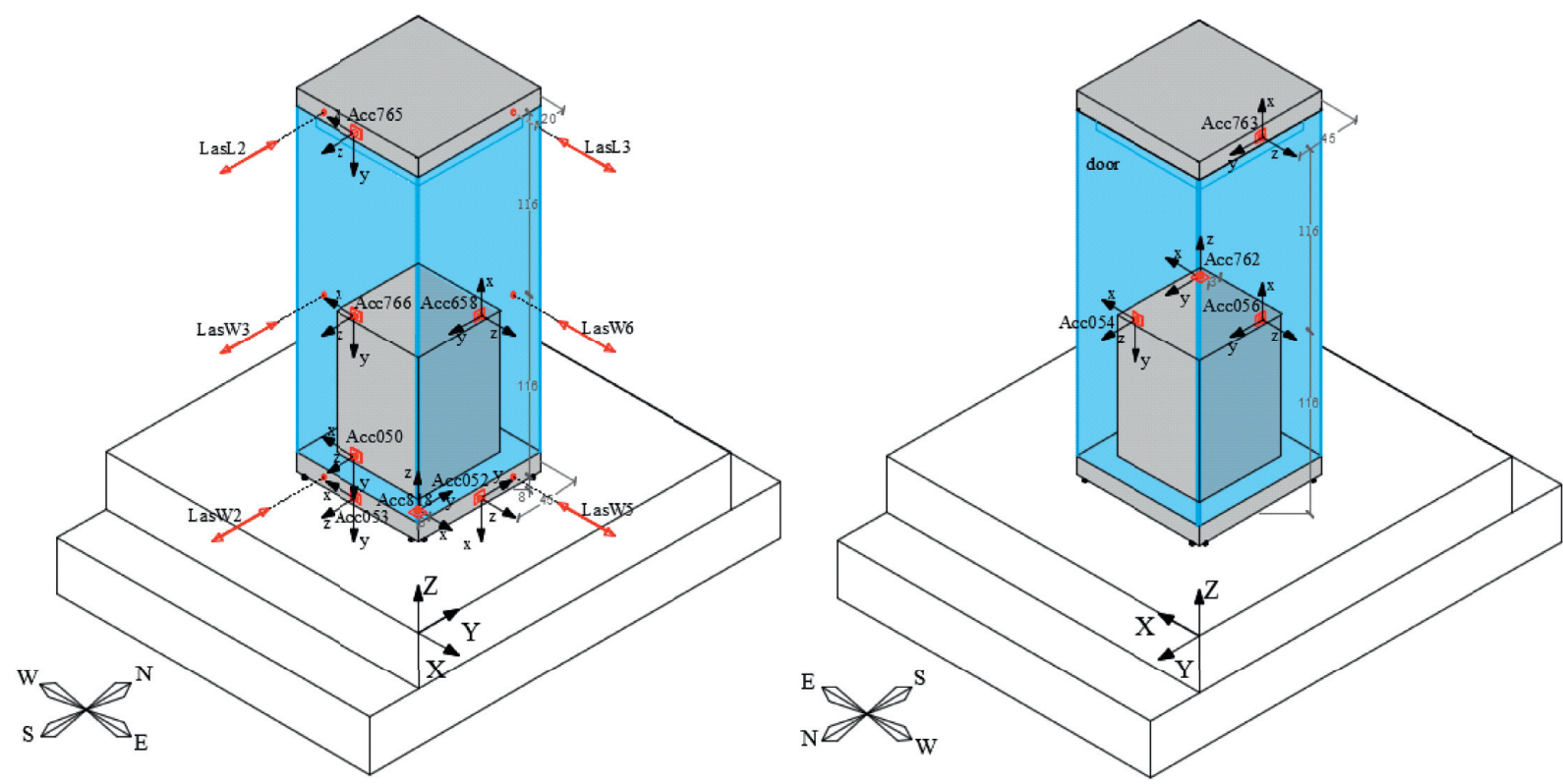

Figure 3: Perspective view of the instrumentation arrangement. The internal shake table accelerometer and laser sensors LasW1 and LasW4 are not depicted.

tests. As these tests were performed considering relatively low intensity shaking levels, the display case did not exhibit rigid motion but only elastic response, behaving as it was fixed at its base. Accordingly, the application of half-power bandwidth method is reasonably consistent. The time history acceleration recorded on both table and display case components was assessed, and the peak values, i.e., PFA and peak component acceleration (PCA), were associated with the incremental test intensities. The component amplification factor, defined as the ratio between PCA and PFA, was computed considering multiple components of the display case and associated with incremental tests. The time history displacement recorded on table and display case was assessed, including both absolute and relative displacement measures; the peak relative displacement is associated with the incremental test intensity.

A preliminary damage assessment was performed considering the following damage states (DSs): DS0 (absent damage), DS1 (minor damage), DS2 (moderate damage), and DS3 (major damage) (e.g., [19, 39]). DS1 achievement implies the need to reposition the specimen or art object in order to restore its original condition; DS2 achievement implies the need to partially replace the components of the specimen; DS3 implies the complete replacement of the specimen, and the life safety is not ensured. Usually, the difference between DS2 and DS3 is due to significant damage that could result in loss of life $[5,49]$. In the case of extremely valuable components (e.g., museum art object), significant motion and/or large residual displacement of the component could even be associated with DS3, even though this response is not associated with loss of life. In fact, this response could result in critical economic and cultural losses. It should be noted that the damage correlations should also depend on the desired level of safety and on importance of the building and that the assessment of museum facilities and art object should follow more conservative rules. The correlation between the exhibited damage and the DS occurrence (namely, damage scheme) was assessed according to past studies [19] and abovementioned consideration. After each shaking level, the damage was observed by inspecting the physical conditions of the components of the display case and art object. The damage level required to reach a given DS was identified for each component of the display case and art object; obviously, the DS is the maximum among the different DSs recorded in each component. In particular, the damage exhibited by the specimens was assessed by both observational checks and data analysis. The observational assessment was carried out during the tests and by checking the recorded videos, whereas a more quantitative assessment was performed through the analysis of the testing output data. The results reported in the following sections should be considered as preliminary findings, and the damage assessment should not be considered exhaustive.

\section{Results and Discussion}

3.1. RRS to TRS Compatibility. Figure 5 depicts the RRS to TRS compatibility related to tests (a) AC01, (b) AC05, and (c) AC09 ( $X$ direction), corresponding to $S_{D S}$ equal to $0.10,0.25$, and $0.45 \mathrm{~g}$, respectively. The recorded (test) TRS were quite similar to the RRS, and the spectrum compatibility was verified for all tests. In particular, even in the only case in which the TRS fall below RRS (i.e., Figure 5(a), corresponding to frequency between 18.5 and $20.8 \mathrm{~Hz}$ ), the compatibility is confirmed since (a) TRS ordinate is not lower than $90 \%$ RRS ordinate and (b) TRS exceed or equal the RRS corresponding to the superior and inferior adjacent sixth of octave [35]. 


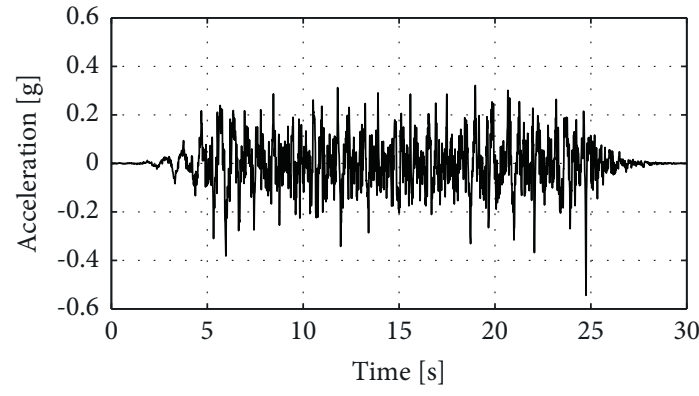

_ Input signal - X direction

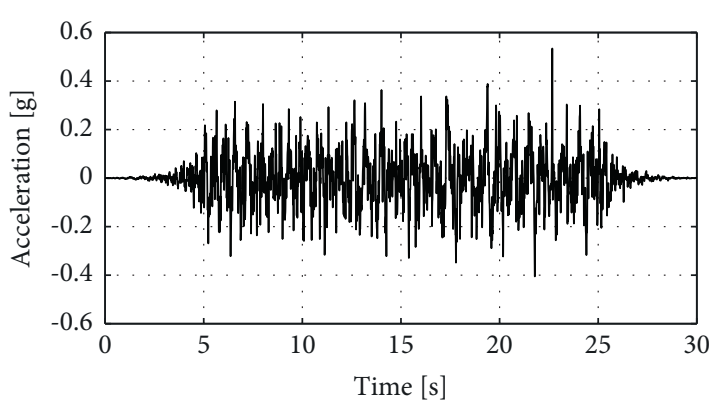

_ Input signal - Y direction

(a)

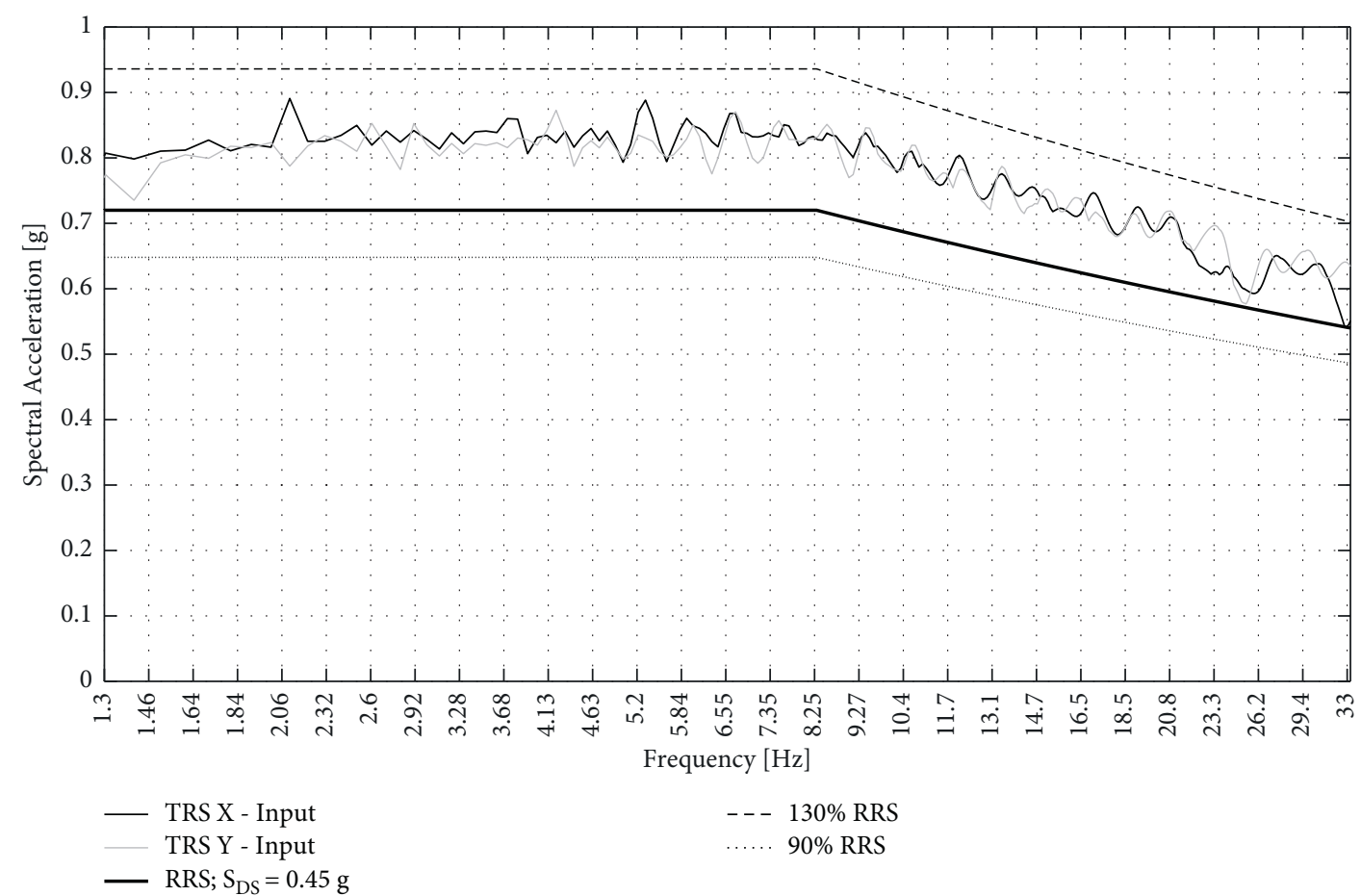

(b)

FIGURE 4: AC156 testing input corresponding to $\mathrm{S}_{\mathrm{DS}}$ equal to $0.45 \mathrm{~g}$ : (a) acceleration time histories and (b) acceleration response spectra (i.e., test response spectra (TRS) and required response spectra (RRS)).

3.2. Dynamic Identification. The dynamic identification results are shown in Figure 6: (a) transfer curves (RAN test curves and median curves); (b) peaks of first fundamental vibration mode; and (c) damping ratios, associated with (1) $X$ and (2) $Y$ directions. In particular, Acc765z and Acc763z were considered as output accelerometers for $X$ and $Y$ directions, respectively, whereas AccT was considered as input accelerometer. RAN1000 and RAN2000 results are not depicted as the tests did not produce consistent transfer functions, and this was reasonable due to the too low intensity amplitude of the random signals (Table 1). It should be noted that the specimen exhibited a complex frequency response (i.e., irregular and multiple vibration modes, Figure 6(a)) and the frequency peaks associated with first modes (Figure 6(b)) cannot fully describe the dynamic properties of the specimen. This points out that the system represents a multiple-degree-of-freedom system. Considering $X$ direction, two sets or families of transfer curves can be identified (Figure 6(a).1): (1) RAN1001, RAN1002, and RAN1006; (2) RAN1003, RAN1004, RAN1005, RAN1007, and RAN1008. Set 1 curves are quite similar to each other and exhibit two significant frequency peaks, corresponding to about $8 \mathrm{~Hz}$ (fundamental frequency) and $13 \mathrm{~Hz}$ and associated with transfer function ordinates equal to about 3.5 and 3.0, respectively. Set 2 curves present fundamental peak frequencies lower than those of Set 1 , corresponding to about $6 \mathrm{~Hz}$, with lower transfer function ordinates; the second peak frequency related to Set 2 curves corresponds to about $18 \mathrm{~Hz}$ and is associated with ordinates equal to about 1. Each curve/frequency set might be associated with a different arrangement/settlement/adjustment condition of the specimen components, activated by the course of the incremental tests. Along $Y$ direction, the transfer curves are 
TABLE 1: Testing program and maximum recorded acceleration: dynamic identification tests (random (RAN) tests) and seismic performance tests (AC156 (AC) tests).

\begin{tabular}{lcccc}
\hline Test ID & Direction(s) & $\begin{array}{c}\mathrm{S}_{\mathrm{DS}} \\
(\mathrm{g})\end{array}$ & $\begin{array}{c}\mathrm{PFA}_{\mathrm{x}} \\
(\mathrm{g})\end{array}$ & $\begin{array}{c}\mathrm{PFA}_{\mathrm{y}} \\
(\mathrm{g})\end{array}$ \\
\hline RAN1000 & $X$ & - & 0.05 & - \\
RAN2000 & $Y$ & - & - & 0.03 \\
AC01 & $X, Y$ & 0.05 & 0.05 & 0.08 \\
AC02 & $X, Y$ & 0.10 & 0.10 & 0.18 \\
RAN1001 & $X$ & - & 0.10 & - \\
RAN2001 & $Y$ & - & - & 0.06 \\
AC03 & $X, Y$ & 0.15 & 0.17 & 0.30 \\
RAN1002 & $X$ & - & 0.10 & - \\
RAN2002 & $Y$ & - & - & 0.08 \\
AC04 & $X, Y$ & 0.20 & 0.24 & 0.38 \\
RAN1003 & $X$ & - & 0.10 & - \\
RAN2003 & $Y$ & - & - & 0.08 \\
AC05 & $X, Y$ & 0.25 & 0.31 & 0.43 \\
RAN1004 & $X$ & - & 0.11 & - \\
RAN2004 & $Y$ & - & - & 0.08 \\
AC06 & $X, Y$ & 0.30 & 0.36 & 0.50 \\
RAN1005 & $X$ & - & 0.11 & - \\
RAN2005 & $Y$ & - & - & 0.08 \\
AC07 & $X, Y$ & 0.35 & 0.42 & 0.54 \\
RAN1006 & $X$ & - & 0.10 & - \\
RAN2006 & $Y$ & - & - & 0.08 \\
AC08 & $X, Y$ & 0.40 & 0.47 & 0.59 \\
RAN1007 & $X$ & - & 0.11 & - \\
RAN2007 & $Y$ & - & - & 0.08 \\
AC09 & $X, Y$ & 0.45 & 0.54 & 0.63 \\
RAN1008 & $X$ & - & 0.10 & - \\
RAN2008 & $Y$ & - & - & 0.07 \\
\hline & & & &
\end{tabular}

more similar to each other, identifying similar fundamental peaks and transfer curve ordinates (Figure 6(a).2). In particular, the fundamental peak frequencies are equal to about $7 \mathrm{~Hz}$ and correspond to transfer curve ordinates equal to about 4 . A second peak frequency can be identified, corresponding to about $11 \mathrm{~Hz}$ and transfer function ordinates ranging between 2 and 2.75 .

The fundamental frequency decreases overall as the incremental tests proceed along the $X$ direction, and the only anomaly is associated with RAN1006 case, where the fundamental frequency exceeds RAN1001 and RAN1002 ones (Figure 6(b).1). Despite this anomaly, it might be hypothesized that the progress of the incremental tests determines deformation/adaptation conditions that, although minor, affect the elastic properties of the specimens in terms of fundamental frequency. In fact, this influence is not actually likely to be associated with damage condition, but with different arrangements of the parts and connections of the specimens. A more regular and gradual decrease in fundamental frequency is observed along the $Y$ direction (Figure 6(b).2). The anomaly related to RAN1006 (Figure 6(b).1) might be caused by a change in arrangement/ settlement/adjustment condition of the specimen due to incremental test preceding test RAN1006. To interpret this anomaly, it should be also recalled that the specimen consists in a complex system of several components connected to each other through noncontrolled constraints, and this is associated with an extremely complex and irregular elastic response. For example, a temporary loosening or tightening condition of an intra- or inter-component connection might have affected the elastic vibration of the component. However, in this preliminary stage, it is not possible to provide more definite comments.

The damping ratio associated with the $X(Y)$ direction ranges from about 16 to $25 \%$ (10-13\%), showing that the specimen has higher damping properties along the $X$ direction. The evolution of the damping ratio over the incremental tests is nonmonotonic; in particular, the ratio increases overall along both $X$ and $Y$ directions, even though the increment related to the $Y$ direction is less significant than that along the $X$ direction. Given the unicity of the tested specimen, it is not meaningful to make quantitative comparisons with literature results. However, the trends and the ranges of the fundamental periods and damping ratios are consistent with previous literature studies (e.g., [44]).

3.3. Acceleration Response. For the sake of brevity, only the time histories related to a representative test are depicted in the paper, whereas peak acceleration related to all tests is shown. Figure 7 depicts the time history acceleration related to test AC05, associated with $\mathrm{S}_{\mathrm{DS}}, \mathrm{PFA}_{\mathrm{x}}$, and $\mathrm{PFA}_{\mathrm{y}}$ equal to $0.25,0.31$, and $0.43 \mathrm{~g}$, respectively. AC05 test is representative since its threshold is between the minor elastic response of the display case (for lower intensities) and the onset of significant rigid motion (from test $\mathrm{AC} 05$ on). In Figure 7, the acceleration is related to both horizontal directions and corresponds to (a) top of the display case, (b) top of pedestal, (c) middle point of representative glass panels, and (d) shake table. In particular, the acceleration of the glass panels is related to their out-of-plane directions. Therefore, $X$ and $Y$ results are associated with different glass panels, as also specified in Figure 7. As a matter of fact, the out-of-plane acceleration of the panels is typically more significant than the in-plane one. Significantly high acceleration was recorded in both directions along the out-ofplane direction of the glass panels (Figure $7(\mathrm{c})$ ), especially considering the shake table acceleration (Figure 7(d)); this proves the considerable acceleration amplification associated with the behavior of the glass panels. The acceleration measured at the top of the display case is also significant, but it is lower than the one recorded on the pedestal. The vase, supported by the pedestal, was subjected to acceleration significantly larger than the shake table one, with extremely high peaks.

Figure 8 shows the peak component acceleration (PCA) evolution over the incremental tests, related to (a) display case top, (b) pedestal top, (c) glass panels middle point, and (d) shake table, along both horizontal directions. PCA evolution related to both display case top (Figure 8(a)) and pedestal (Figure $8(\mathrm{~b})$ ) is more regular than the one recorded on the glass panels (Figure $8(\mathrm{c})$ ). In the former cases (Figures 8(a) and $8(\mathrm{~b}))$, there is an increase over the incremental tests that is relatively similar to the one associated with the accelerograms recorded on the shake table (Figure 8(d)). The acceleration peaks related the glass panels (Figure $8(\mathrm{c})$ ) are more irregular 


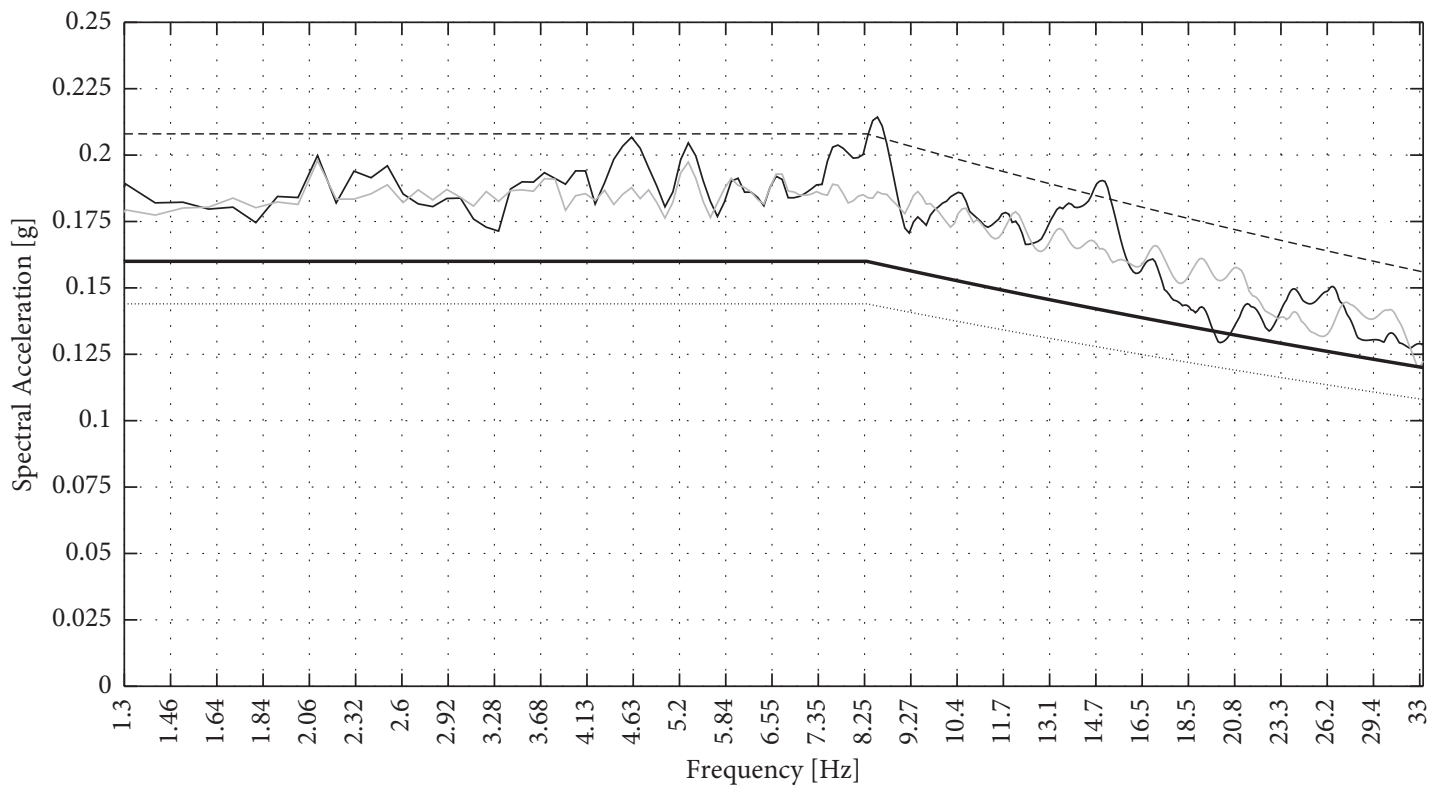

— TRS X - Test

TRS X - Input

-- $130 \%$ RRS

— RRS; $\mathrm{S}_{\mathrm{DS}}=0.10 \mathrm{~g}$

(a)

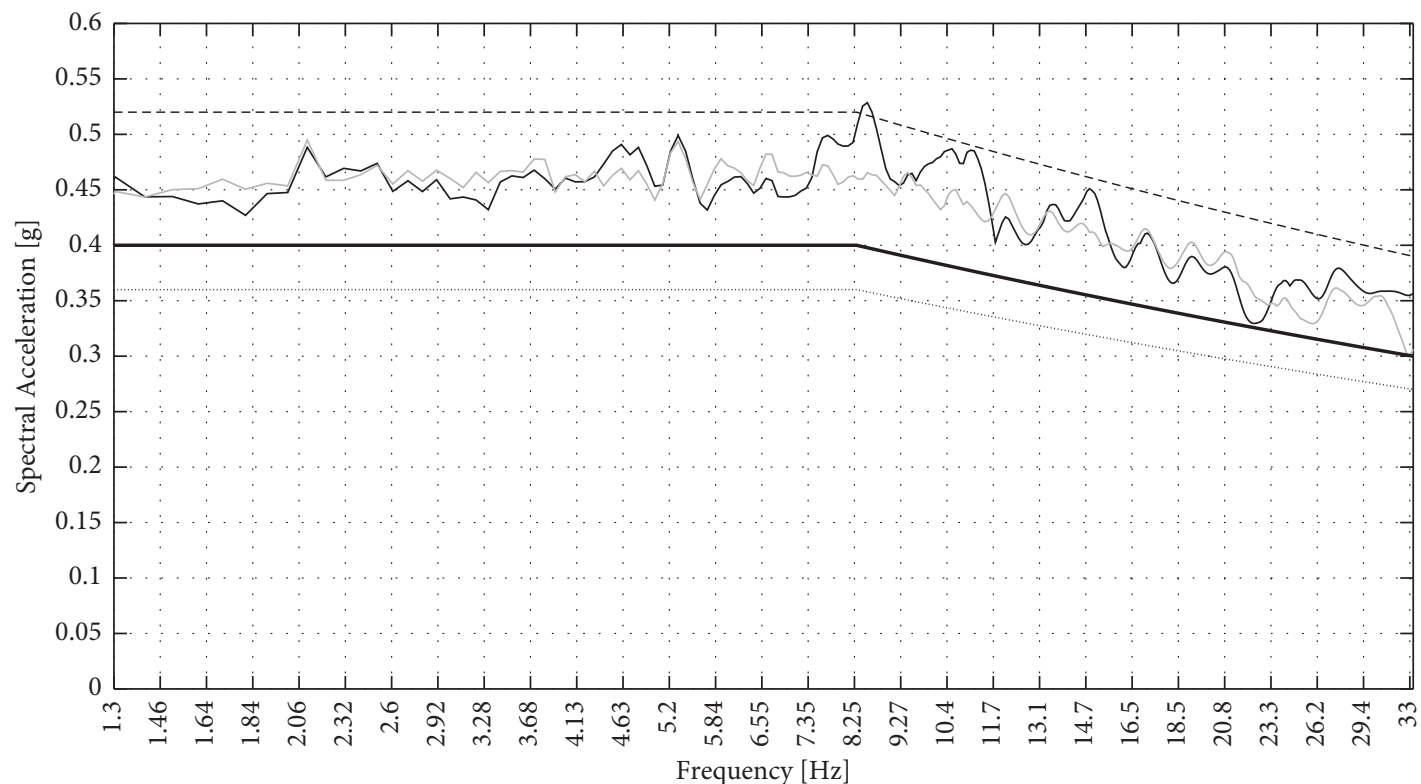

TRS X - Test

TRS X - Input

- - $130 \%$ RRS

RRS; $S_{D S}=0.25$

(b)

Figure 5: Continued. 


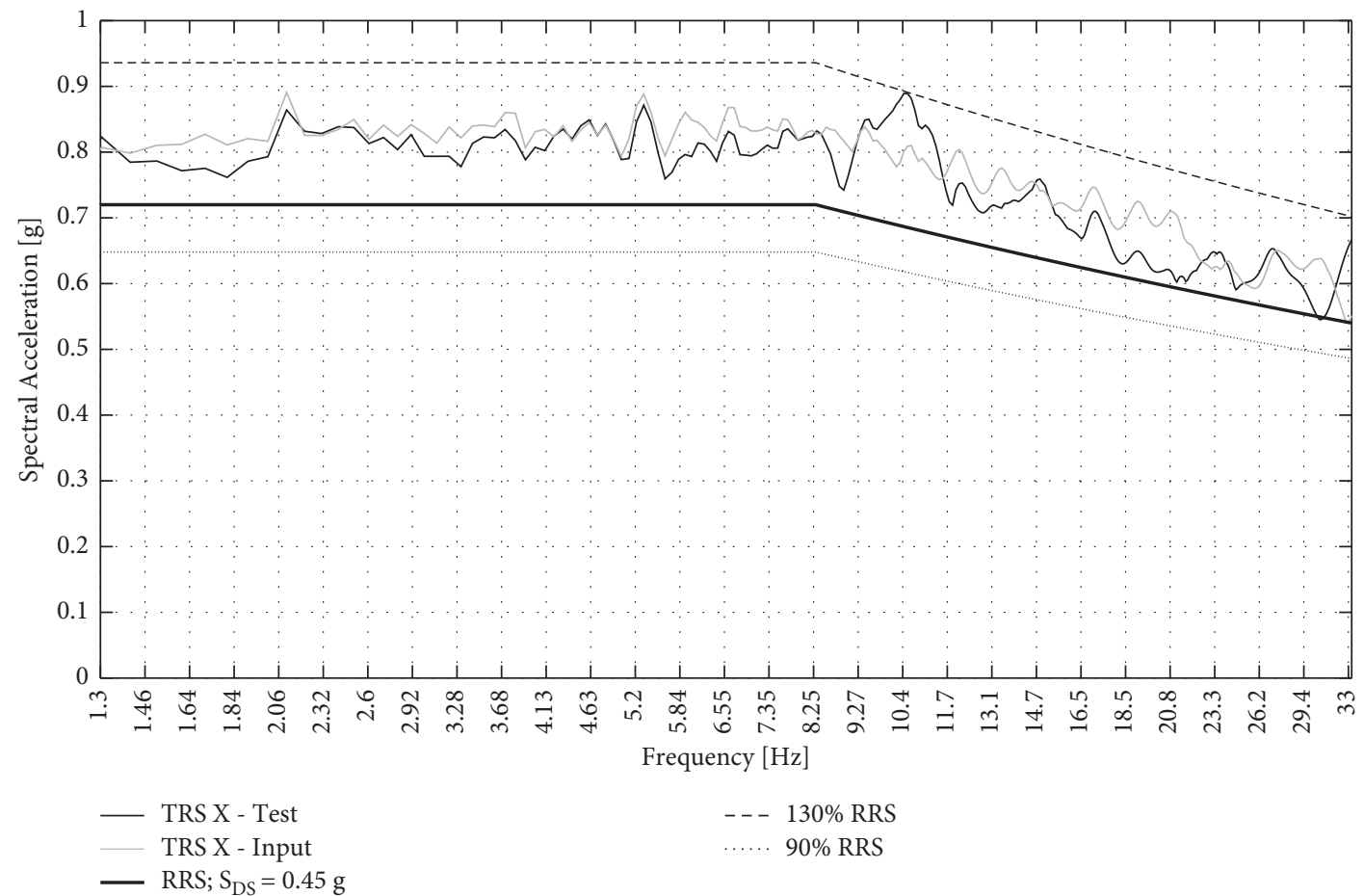

(c)

FIGURE 5: Spectrum-compatibility analysis: required response spectra (RRS) and test response spectra (TRS) along the $X$ direction. The results are related to tests: (a) AC02 ( $\mathrm{S}_{\mathrm{DS}}$ equal to $0.10 \mathrm{~g}$ ), (b) AC05 ( $\mathrm{S}_{\mathrm{DS}}$ equal to $0.25 \mathrm{~g}$ ), and (c) AC09 ( $\mathrm{S}_{\mathrm{DS}}$ equal to $0.45 \mathrm{~g}$ ). "TRS X - Test" and TRS X - Input" spectra correspond to recorded test signal and assigned input signal.

as the testing intensity grows (Figure $8(\mathrm{~d})$ ). Considering both display case top and pedestal top accelerometer, the acceleration peaks related to the $Y$ direction are higher than the ones associated with the $X$ direction, similarly to the shake table acceleration peaks, whereas, for glass panel acceleration, the peaks related to the horizontal directions are overall more similar to each other. The glass acceleration peaks are significantly higher than the ones recorded on the other components. In some cases, extremely high acceleration (e.g., much greater than $1.0 \mathrm{~g}$ ) peaks were recorded on the components; these peaks were determined by a significant interaction between the monitored component and the adjacent parts (i.e., test AC08 on glass panel and test AC09 on pedestal). In particular, the significant out-of-plane motion of the glass panels and the hammering against the adjacent (perpendicular) glass panels determined extremely high and sudden acceleration peaks. These responses were not considered in a quantitative manner in this study.

The evolution of the component amplification factor over the PFA is depicted in Figure 9, corresponding to (a) display case top, (b) pedestal top, and (c) glass panels middle point, along both horizontal directions. The PFA is considered as abscissa axis since $X$ and $Y$ PFA are relatively different over the same AC tests (Figure 8(d)); accordingly, the influence of the intensity can be directly assessed on component amplification factor. The trend of the component amplification factor over the incremental tests is similar over the different components of the display case, even though significant ordinate values are observed among the different components, especially along $X$ direction. Overall, the component amplification factor along the $X$ direction is larger than that along the $Y$ direction over low intensity tests, especially for glass panel (Figure 9(c)) recording.

The maximum component amplification is always associated with test AC02 (PFA equal to about $0.10 \mathrm{~g}$ ), whereas the minimum values are related to highest intensity tests. This points out that the most significant elastic response of the display case components is associated with about $0.10 \mathrm{~g}$ PFA intensities, when the specimen does not exhibit rigid motion, whereas the less significant elastic response is related to tests in which the specimen exhibits a significant rigid motion. It is recalled that dynamic identification tests were performed considering this intensity, to maximize the relevance of the elastic vibrational response. The maximum component amplification related to display case top (Figure 9(a)) and pedestal top (Figure 9(b)) is overall lower than typical values recommended by building codes (e.g., 2.5 according to ASCE 7-16 [43]), especially the ones related to display case top. Conversely, maximum component amplification factor on the glass panels (Figure 9(c)) (e.g., equal to about 5 for $X$ direction and $\mathrm{AC} 02$ test) is larger than these values, especially over low intensities.

3.4. Displacement Response. The displacement time histories related to AC05 test are depicted in Figure 10: (a) absolute displacement of the display case top, (b) absolute displacement of the shake table, (c) relative displacement 

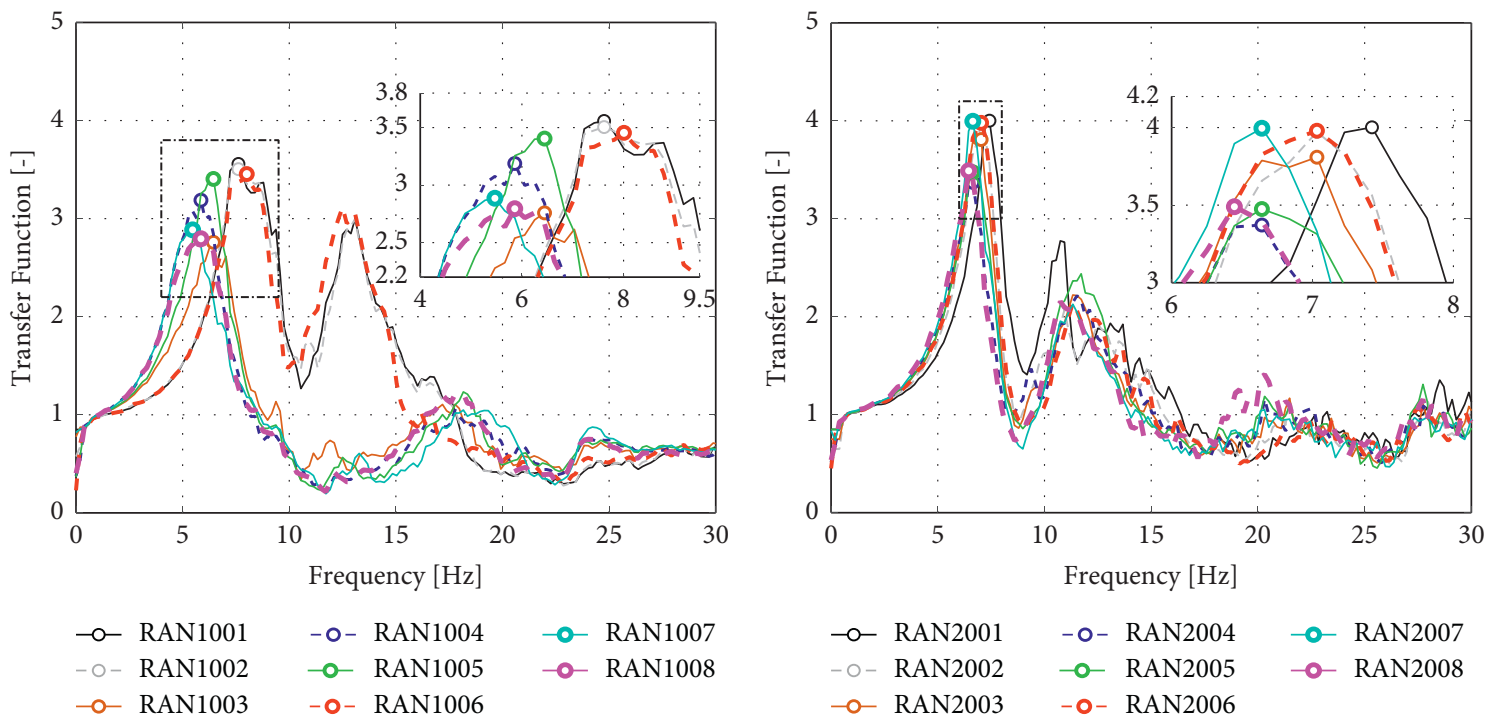

(a)
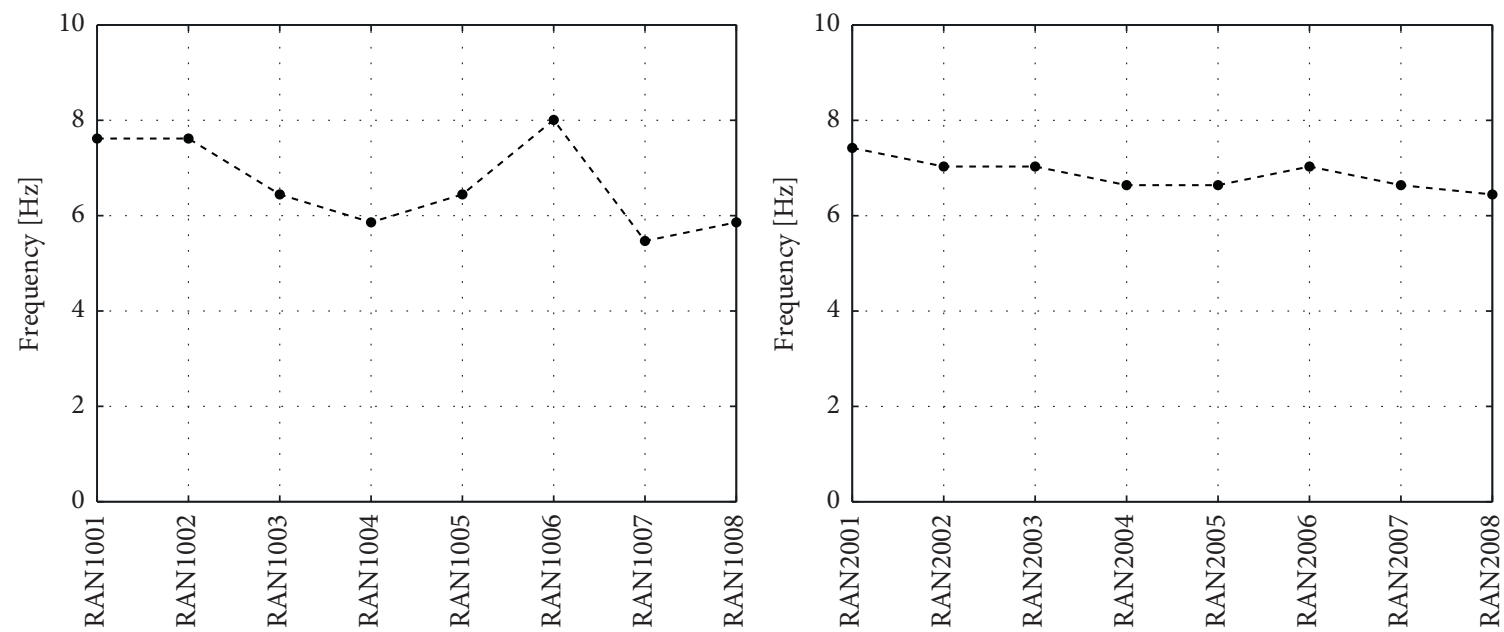

(b)
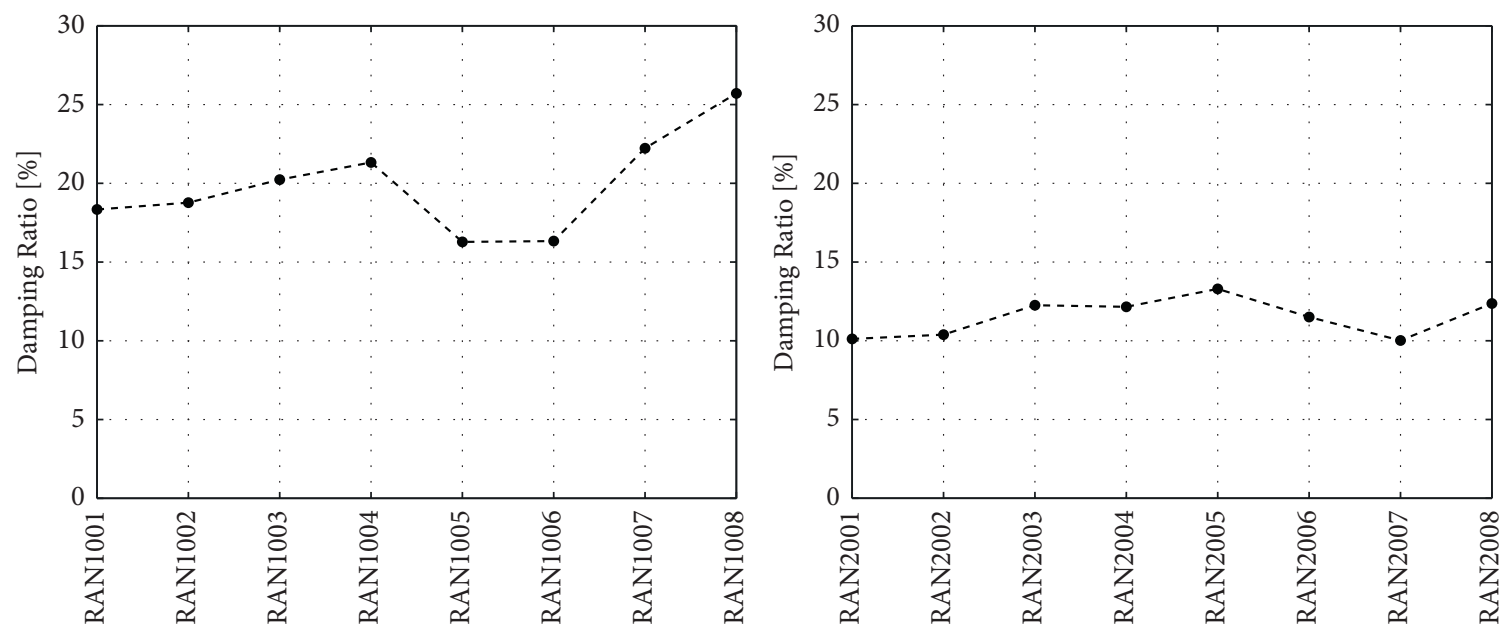

(c)

FIgURE 6: Dynamic identification: (a) transfer curves related to all dynamic identification (RAN) tests, (b) the frequency peaks associated with first vibration mode, and (c) damping ratios evolution over RAN tests, estimated along (1) $X$ and (2) $Y$ directions. 


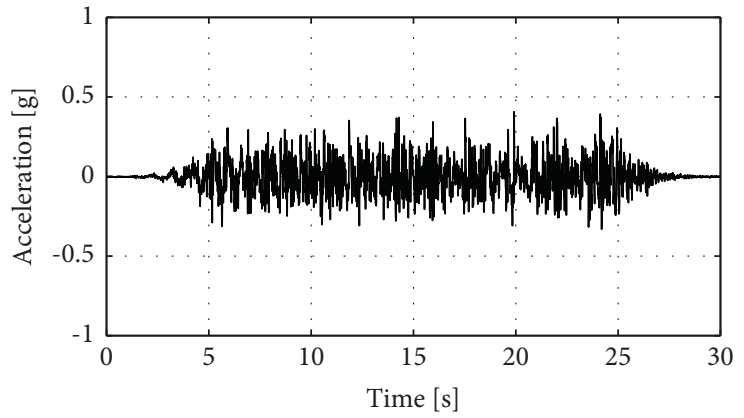

- Acc $765 \mathrm{x}$

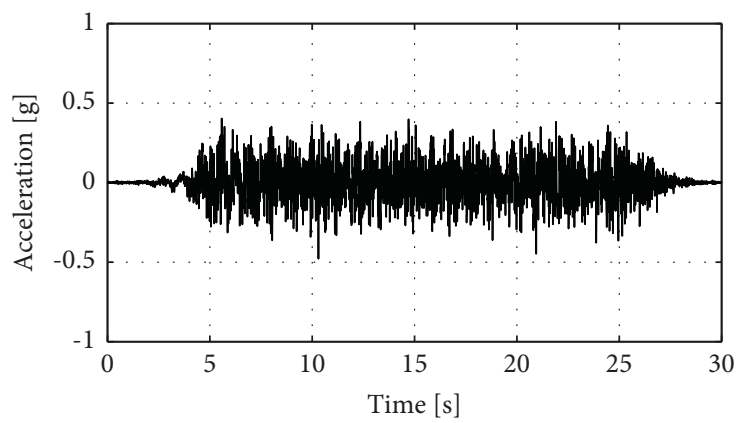

- Acc762x

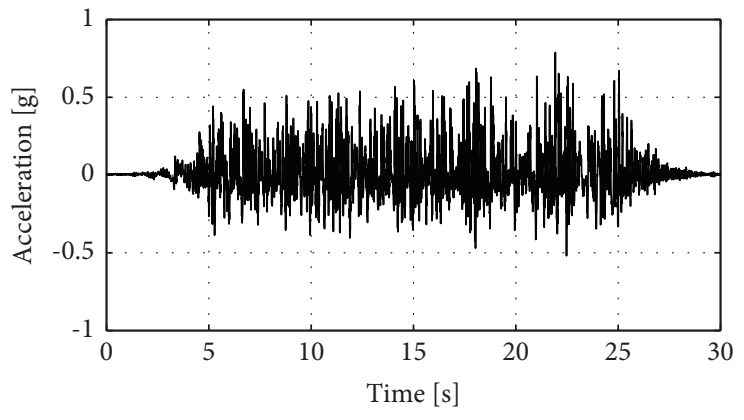

- Acc658z

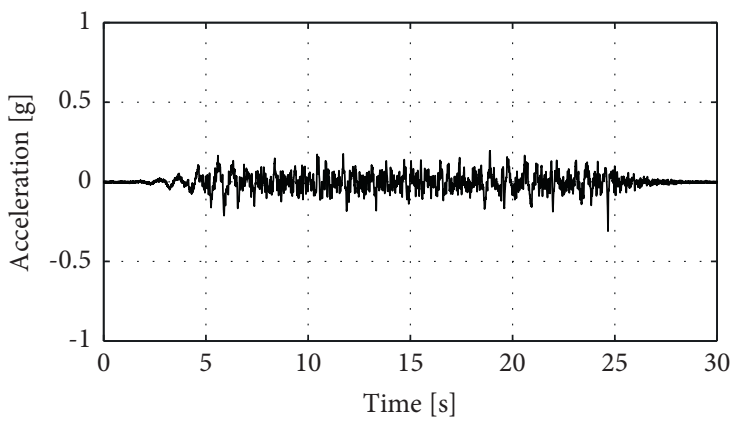

- AccTX

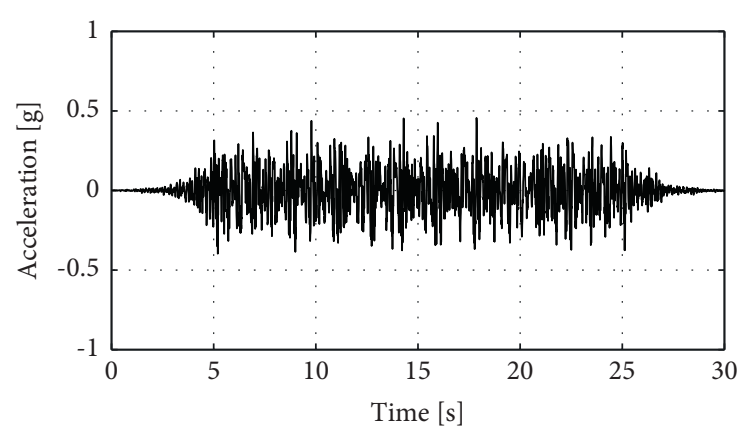

- Acc765z

(a)

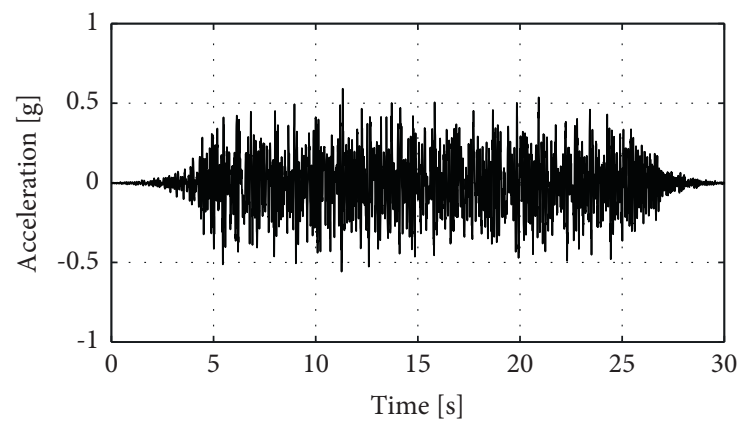

(b)

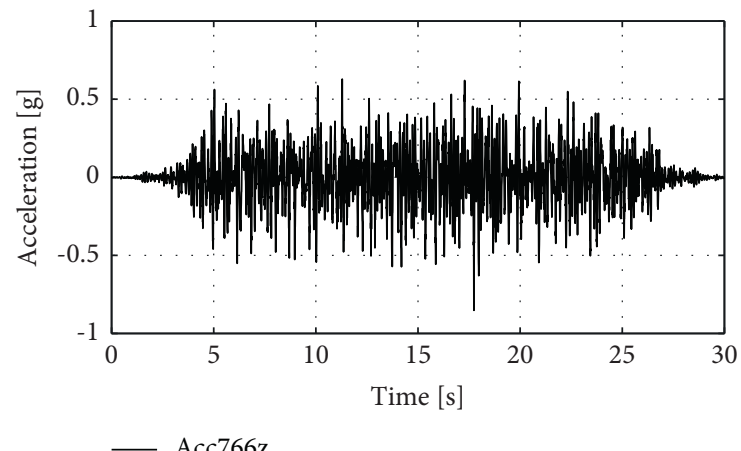

(c)

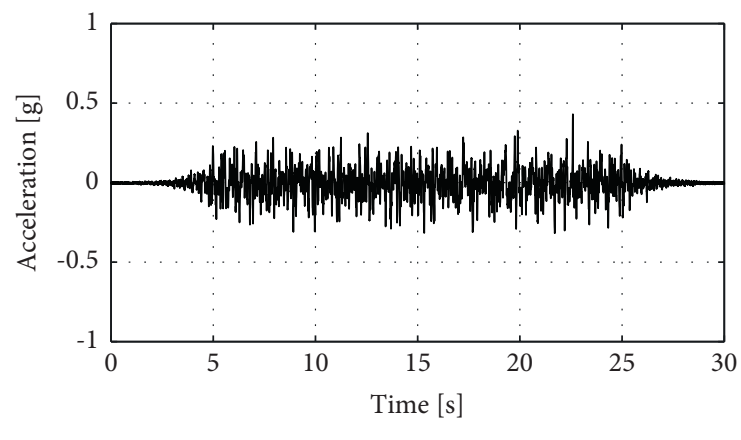

(d)

FIgURE 7: Acceleration time histories related to AC05 test, corresponding to (a) display case top, (b) pedestal top, (c) glass panels middle point, and (d) shake table, along (1) $X$ and (2) $Y$ directions. 


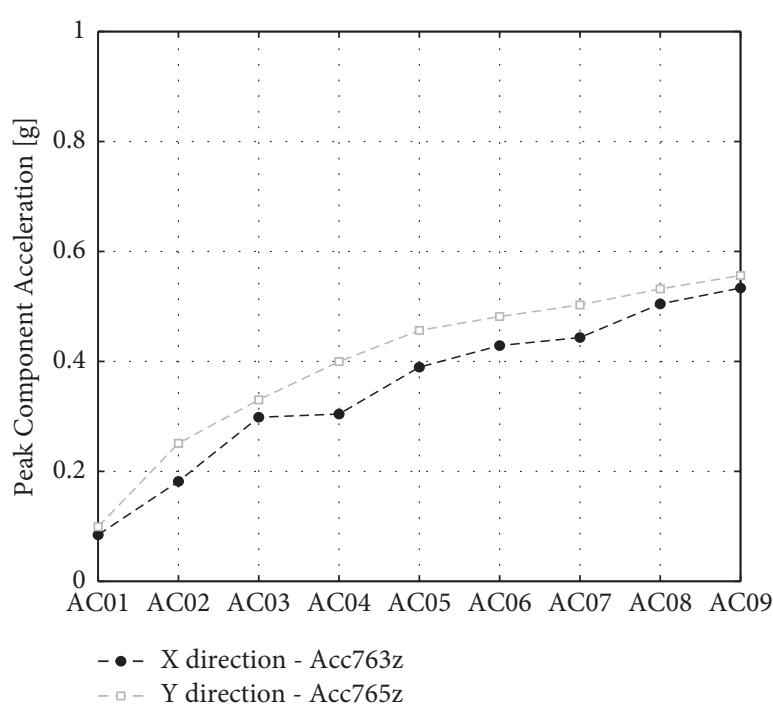

(a)

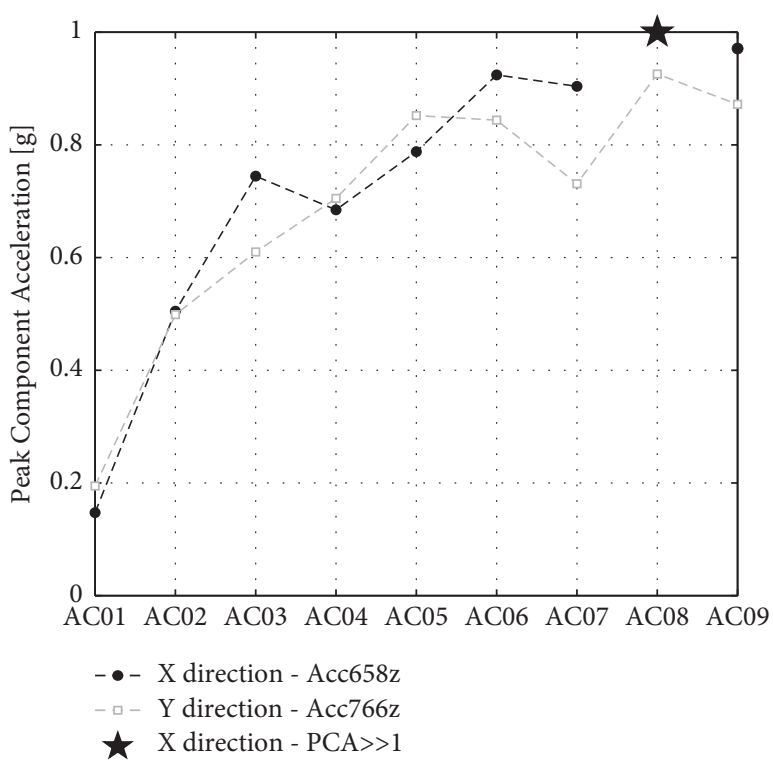

(c)

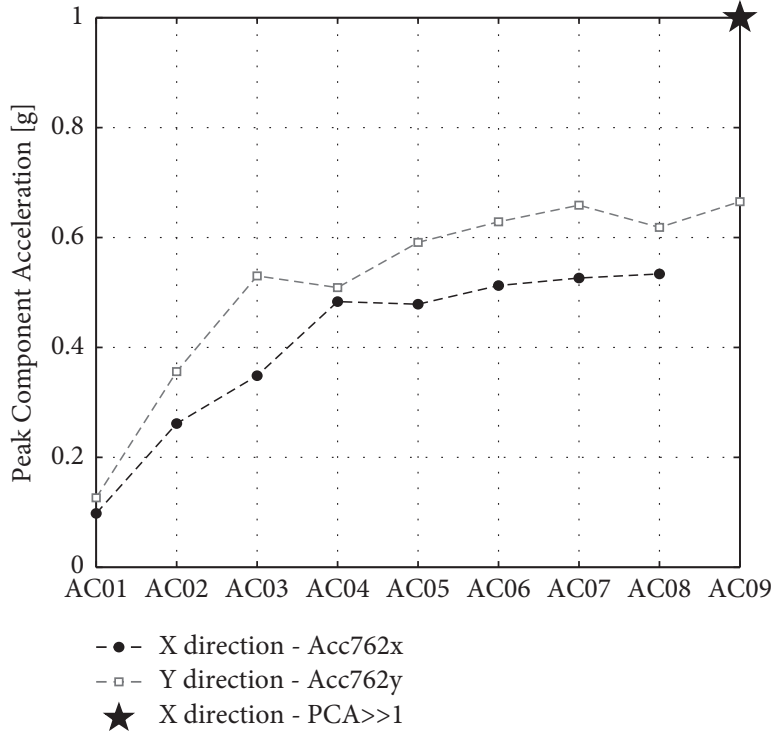

(b)

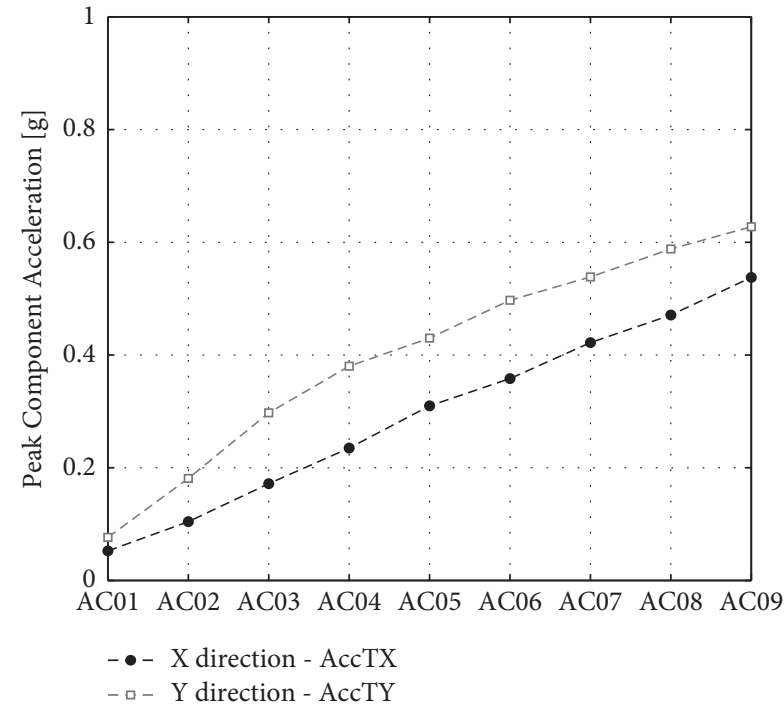

(d)

FIgURE 8: Peak component acceleration (PCA) evolution over the seismic performance tests corresponding to (a) display case top, (b) pedestal top, (c) glass panels middle point, and (d) shake table, along both horizontal directions.

of the display case assessed between display case top and shake table (L3-W4 along $X$ direction and L2-W1 along $Y$ direction), and (d) relative displacement of the display case assessed between display case top and bottom (L3-W5 along $X$ direction and L2-W2 along $Y$ direction). The display case exhibited a complex (rigid) rotationaltranslational motion, in addition to the minor elastic response. The response of the display case started to become more significant and more complex after the first 5-7 seconds, which correspond to the end of the triggering part and the onset of the strong motion part of the acceleration input. This can be derived by comparing the absolute displacement at the display case top (Figure 10(a)) and the shake table displacement
(Figure $10(\mathrm{~b})$ ) or by assessing the relative displacement (Figure 10(c)). In particular, the rotational-oscillatory and translational contribution of the case motion can be clearly observed in Figure 10(c). After 5-7 seconds, the time history begins to deviate from the zero-displacement axis. This deviation results in significant residual displacement of the display case in both directions (i.e., about -21 and $+26 \mathrm{~mm}$ along the $X$ and $Y$ directions, respectively). The rotational-oscillatory motion of the specimen can be identified in Figure 10(d), as the depicted relative displacement accounts for the horizontal projection of the inclination of the display case, which is associated with rotational-oscillatory motion. This relative displacement is not significantly large if 


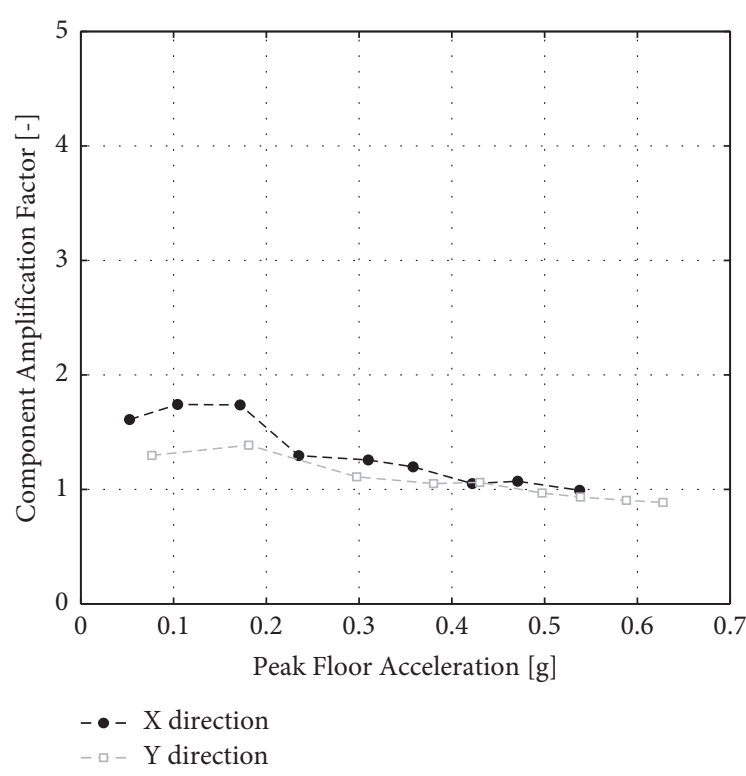

(a)

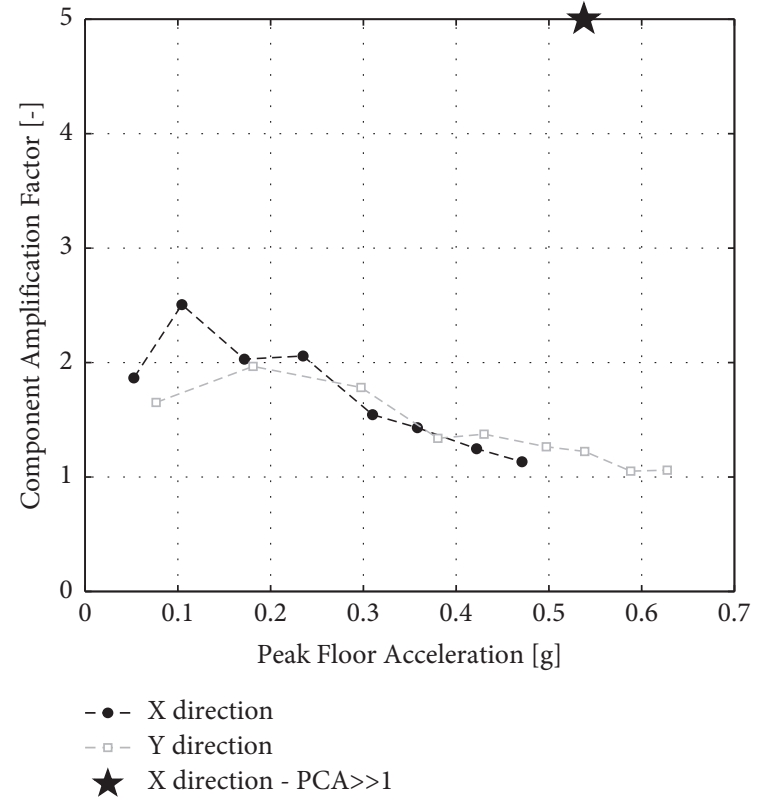

(b)

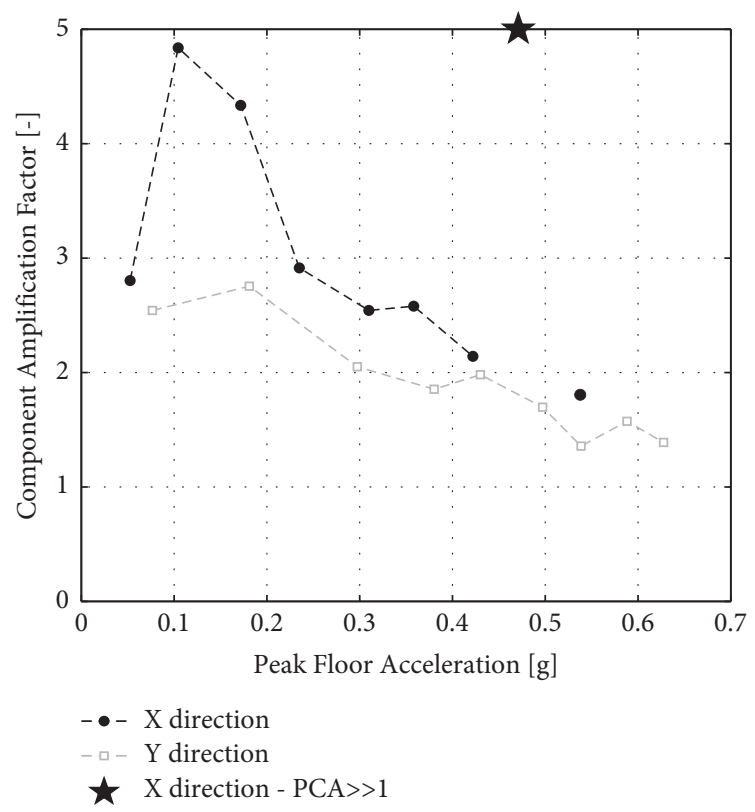

(c)

Figure 9: Component amplification factor evolution over the seismic performance tests corresponding to (a) display case top, (b) pedestal top, and (c) glass panels middle point, along both horizontal directions.

compared with the one associated with translational motion (Figure 10(c)). It can be concluded that the sliding response is more significant than the rocking one.

In order to assess the significance of the rotationaloscillatory motion over the incremental tests, the peak relative displacement evaluated between top and base of the display case is shown in Figure 11; AC09 $Y$ result is not depicted in Figure 11 as one of the lasers went out of scale. The peak relative displacement is comparable along the $X$ and $Y$ directions and approximately increases as the test intensity grows. Even for significant testing intensities, the peak relative displacement is not critically large, especially if it is compared with relative displacement between display case top and shake table and with height of the specimen. This stresses the relevance of the sliding response of the display case over the rocking one.

3.5. Preliminary Damage Assessment. Prior to presenting the damage assessment results, it is recalled that this study was not aimed at assessing in a quantitative and accurate manner the damage exhibited by both display case and vase. 


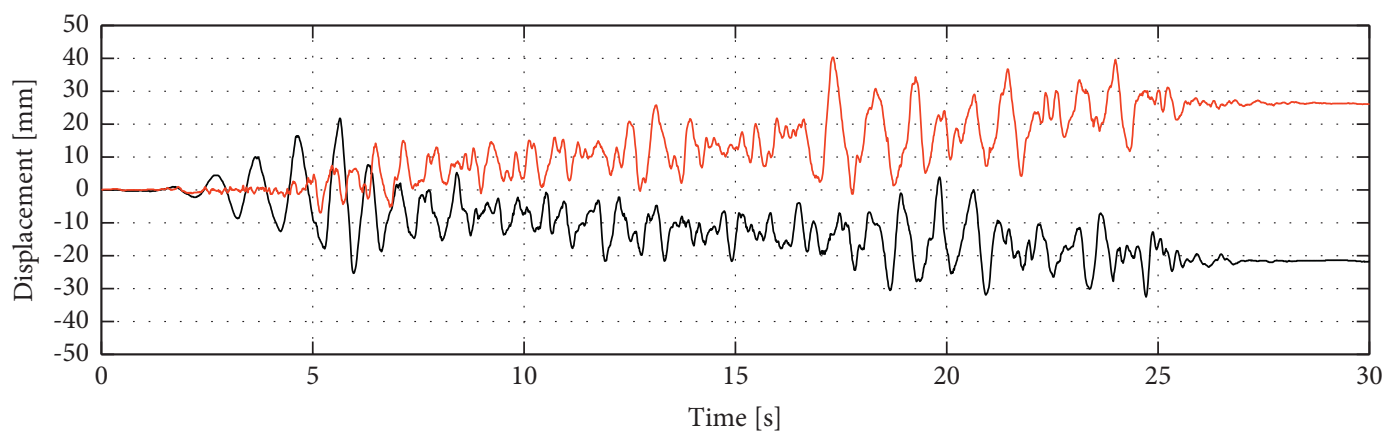

_ X direction - LasL3

_ Y direction - LasL2

(a)

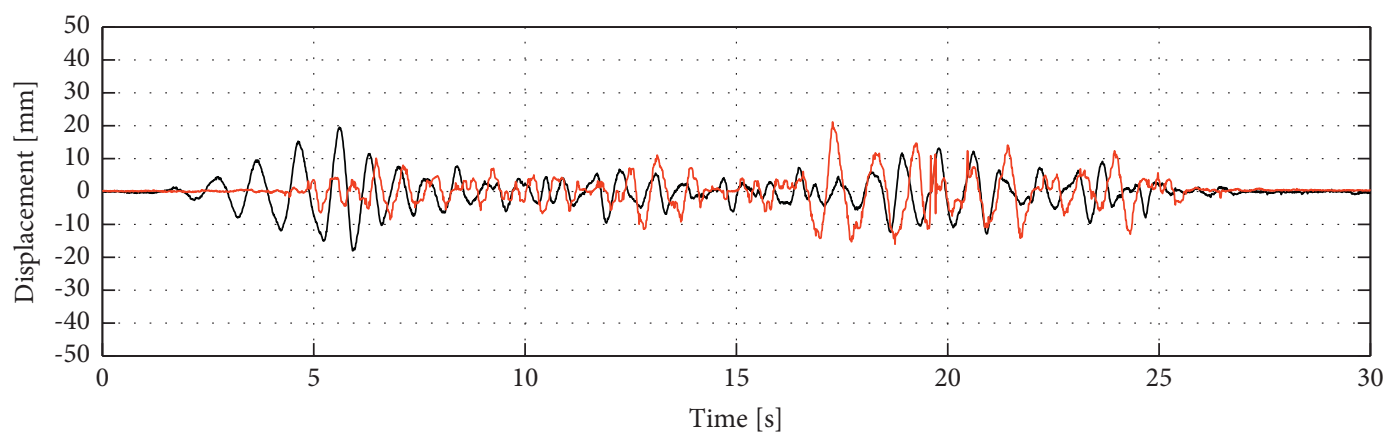

- X direction - LasW4

_ Y direction - LasW1

(b)

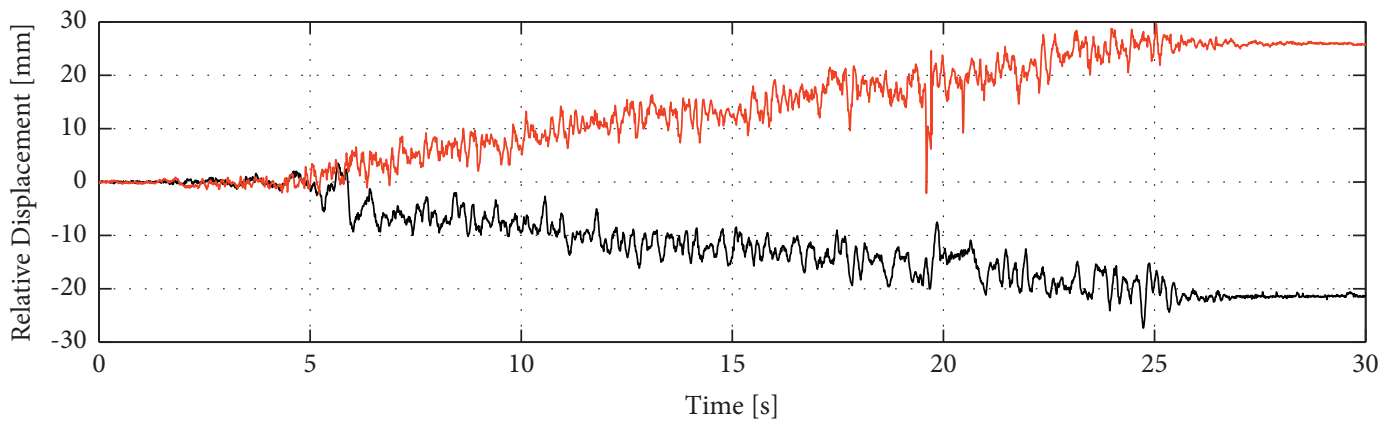

— X direction - L3-W4

$\mathrm{Y}$ direction - L2-W1

(c)

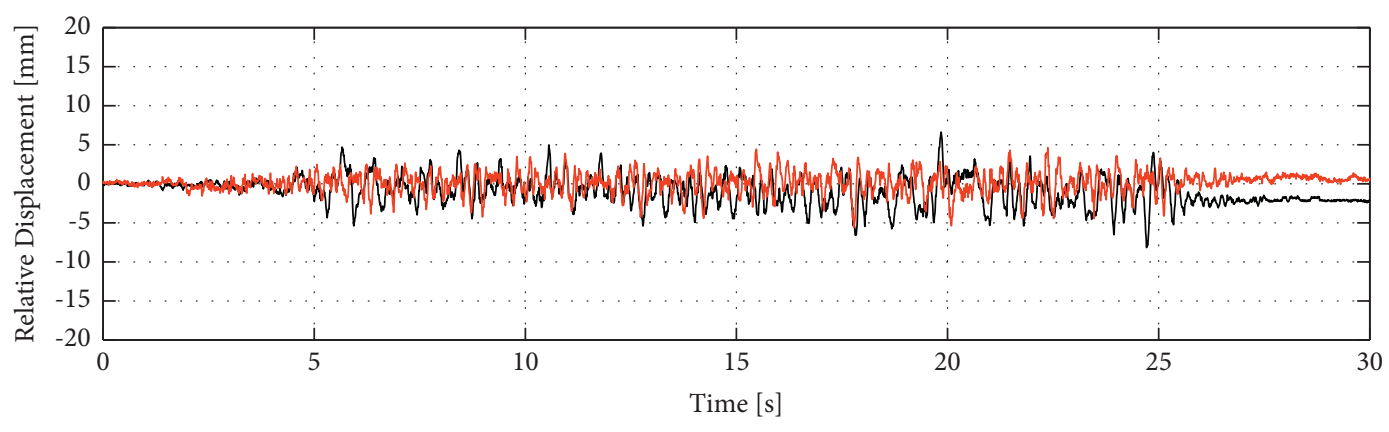

— X direction - L3-W5

— Y direction - L2-W2

(d)

FIGURE 10: Displacement time histories related to AC05 test: (a) absolute displacement of display case top, (b) absolute displacement of shake table, (c) relative displacement of the display case assessed between display case top and shake table (L3-W4 along $X$ direction and L2-W1 along $Y$ direction), and (d) relative displacement of the display case assessed between display case top and bottom (L3-W5 along $X$ direction and L2-W2 along $Y$ direction). 


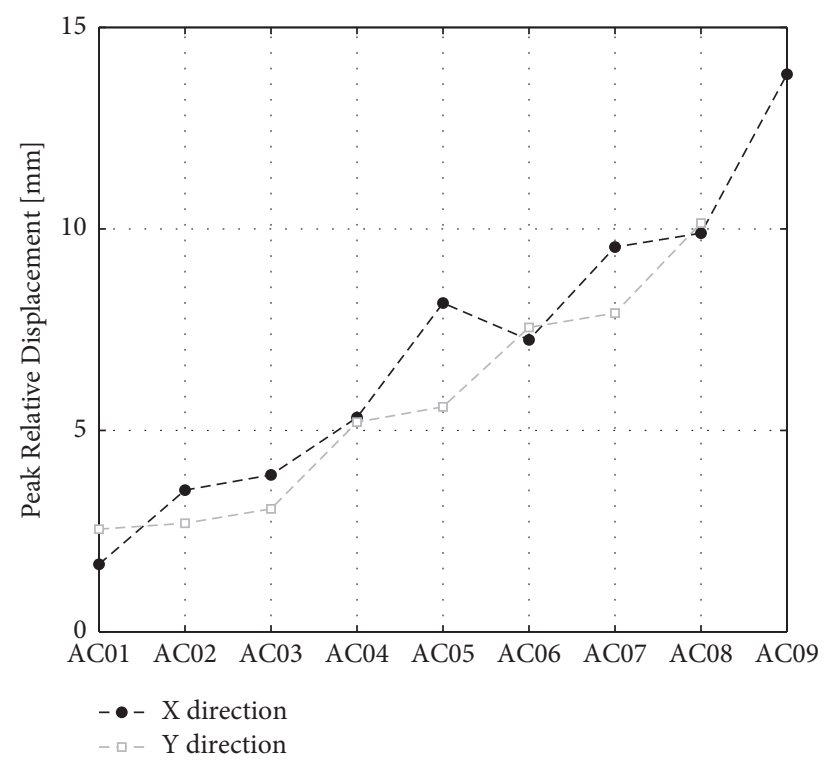

Figure 11: Peak relative displacement evolution over the seismic performance tests, evaluated between the top and base of the display case (L3-W5 along $X$ direction and L2-W2 along $Y$ direction).

Therefore, the following assessment results should be considered as preliminary and tentative estimations, only aiming to shed light on the critical behavior of the tested specimens. A tentative correlation between the seismic tests and the occurring DSs is reported in Table 2, including preliminary information regarding the component motion and the residual displacement. Moreover, the correlation between an engineering demand parameter (EDP) and the occurring DSs is presented. Two EDPs were chosen: PFA and PCA (recorded on the top of the display case). The damage identification reported in Table 2 is based on the assessment of two component response parameters, i.e., component motion and residual displacement, related to each seismic performance test. In particular, three levels of severity are defined for each parameter, i.e., minor, moderate, and major. The occurring DS related to each test is identified according to the detected levels of severity and assuming that the art object has an extremely significant economic and cultural value.

The damage assessment associated with AC01, AC02, and $\mathrm{AC} 03$ resulted in DS0. In particular, the specimen did not exhibit appreciable motion and damage, except for the glass panels, which exhibited a minor to moderate out-ofplane motion (oscillation) during the shaking; minor to moderate glass motion does not reasonably produce any damage or disruption. AC04 test excitation determined minor to moderate motion of the components, with minor residual displacement of the vase. Accordingly, DS1 was assumed to be achieved. In the context of valuable items, even minor motion or residual displacement can be considered to be associated with DS1. For higher intensities, i.e., for AC05 to AC09 test, major component motion and major residual displacement were observed. The glass panels strongly hammered against the adjacent perpendicular glass panels, producing significant impacts. The art object exhibited a major sliding motion from AC05 test on (included), resulting in residual displacement comparable with the semi-dimension of the pedestal's top support surface. However, the object did not fall from the pedestal or hammer against the glass panels. No significant permanent damage/deformation of vase or display case components was observed. Therefore, focusing on the specific and peculiar outcome of the present test, we could reasonably assume DS2. However, in the context of valuable components as art objects, the responses corresponding to AC05 to AC09 tests (i.e., significant motion and large residual displacement) could be reasonably associated with DS3. In fact, a slightly different arrangement or a less stable or more fragile art object could have potentially been damaged by the significant motion observed in the course of these tests: this would have caused extremely significant economic and cultural losses, also compatible with DS3 achievement. Therefore, both DS2 and DS3 are associated with AC05 to AC09 tests, according to the abovementioned motivations and given that the damage scheme also depends on desired level of safety and building/component type/importance. Finally, the authors recall that the damage assessment results are meant to be preliminary and not exhaustive. 
TABLe 2: Preliminary damage assessment.

\begin{tabular}{|c|c|c|c|c|c|c|c|c|c|c|}
\hline \multirow{2}{*}{ Test ID } & \multirow{2}{*}{$\begin{array}{c}\operatorname{Min}\left(\mathrm{PFA}_{\mathrm{x}}, \mathrm{PFA}_{\mathrm{y}}\right) \\
(\mathrm{g})\end{array}$} & \multirow{2}{*}{$\begin{array}{c}\operatorname{Min}\left(\mathrm{PCA}_{\mathrm{x}}, \mathrm{PCA}_{\mathrm{y}}\right) \\
(\mathrm{g})\end{array}$} & \multirow{2}{*}{ DS } & \multicolumn{4}{|c|}{ Component motion } & \multicolumn{3}{|c|}{ Residual displacement } \\
\hline & & & & Display case & Pedestal & Glass & Vase & Display case & Pedestal & Vase \\
\hline AC01 & 0.05 & 0.09 & DS0 & - & - & - & - & - & - & - \\
\hline $\mathrm{AC} 02$ & 0.10 & 0.18 & DS0 & - & - & $*$ & & - & - & - \\
\hline $\mathrm{AC} 03$ & 0.17 & 0.30 & DS0 & - & - & $* *$ & - & - & - & - \\
\hline $\mathrm{AC} 04$ & 0.24 & 0.30 & DS1 & $*$ & $*$ & $* *$ & $*$ & - & - & $*$ \\
\hline $\mathrm{AC} 05$ & 0.31 & 0.39 & $\mathrm{DS} 2 / \mathrm{DS} 3$ & $* * *$ & * & $* * *$ & $* * *$ & $*$ & - & $* * *$ \\
\hline AC06 & 0.36 & 0.43 & $\mathrm{DS} 2 / \mathrm{DS} 3$ & $* * *$ & $*$ & $* * *$ & $* * *$ & $* * *$ & - & $* * *$ \\
\hline $\mathrm{AC} 07$ & 0.42 & 0.44 & $\mathrm{DS} 2 / \mathrm{DS} 3$ & $* * *$ & $* * *$ & $* * *$ & $* * *$ & $* * *$ & $* * *$ & $* * *$ \\
\hline AC08 & 0.47 & 0.51 & $\mathrm{DS} 2 / \mathrm{DS} 3$ & $* * *$ & $* * *$ & $* * *$ & $* * *$ & $* * *$ & $* * *$ & $* * *$ \\
\hline AC09 & 0.54 & 0.53 & $\mathrm{DS} 2 / \mathrm{DS} 3$ & $* * *$ & $* * *$ & $* * *$ & $* * *$ & $* * *$ & $* * *$ & $* * *$ \\
\hline
\end{tabular}

\section{Conclusions}

The paper reported the preliminary results of an experimental campaign aimed at assessing the dynamic properties and the seismic performance of museum objects and artefacts. A typical museum display case was tested on shake table, including the presence of a representative art object (ceramic vase). The main dynamic properties of the specimen were estimated, and both acceleration and displacement recorded on the specimen were assessed, estimating the peak values. Rocking and sliding response of the display case were identified, and component amplification factors associated with both display case and pedestal were assessed. A tentative damage assessment of both display case and vase was carried out.

The study highlights the critical response of the tested display case and vase and stresses the need for further studies investigating the seismic performance of valuable freestanding systems and objects. Further studies should be performed to assess the performance of museum objects through a performance-based engineering approach, also considering analytical and numerical methods. Moreover, fragility and vulnerability curves should be also assessed to provide useful tools for expeditious assessment.

\section{Data Availability}

The experimental and analytical data used to support the findings of this study are available from the corresponding author upon request.

\section{Conflicts of Interest}

The authors declare that there are no conflicts of interest regarding the publication of this paper.

\section{Acknowledgments}

The effort and the support of the Director of MANN, Dr. Paolo Giulierini, are fully acknowledged. Prof. Giuseppe Maddaloni is thanked for his support regarding the experimental testing procedure. The experimental tests were carried out in the framework of the agreement between the National Archaeological Museum of Naples (MANN, Italy) and the Department of Structures for Engineering and Architecture (DIST) (University of Naples Federico II, Italy), i.e., Convenzione MANN-DIST.

\section{References}

[1] D. D’Angela, G. Magliulo, and E. Cosenza, "Towards a reliable seismic assessment of rocking components," Engineering Structures, vol. 230, Article ID 111673, 2021.

[2] E. G. Dimitrakopoulos and T. S. Paraskeva, "Dimensionless fragility curves for rocking response to near-fault excitations," Earthquake Engineering \& Structural Dynamics, vol. 44, no. 12, pp. 2015-2033, 2015.

[3] K. Klaboe, S. Pujol, and L. Laughery, "Seismic response of rocking blocks," Earthquake Spectra, vol. 34, no. 3, pp. 1051-1063, 2018.

[4] M. D. Purvance, A. Anooshehpoor, and J. N. Brune, "Freestanding block overturning fragilities: numerical simulation and experimental validation," Earthquake Engineering \& Structural Dynamics, vol. 37, no. 5, pp. 791-808, 2008.

[5] S. Taghavi and E. Miranda, "Response Assessment of Nonstructural Building Elements. PEER Report 2003/05," Pacific Earthquake Engineering Research Center, University of California at Berkeley, Berkeley, California, 2003.

[6] E. A. Fierro, E. Miranda, and C. L. Perry, Behavior of Nonstructural Components in Recent Earthquakes, American Society of Civil Engineers, Reston, VA, USA, pp. 369-377, 2011.

[7] N. Achour, M. Miyajima, M. Kitaura, and A. Price, "Earthquake-induced structural and nonstructural damage in hospitals," Earthquake Spectra, vol. 27, no. 3, pp. 617-634, 2011.

[8] B. Yön, O. Onat, and M. E. Öncü, "Earthquake damage to nonstructural elements of reinforced concrete buildings during 2011 van seismic sequence," Journal of Performance of Constructed Facilities, vol. 33, Article ID 4019075, 2019.

[9] L. Di Sarno, G. Magliulo, D. D’Angela, and E. Cosenza, "Experimental assessment of the seismic performance of hospital cabinets using shake table testing," Earthquake Engineering \& Structural Dynamics, vol. 48, no. 1, pp. 103-123, 2019.

[10] D. Konstantinidis and N. Makris, "Experimental and analytical studies on the response of freestanding laboratory equipment to earthquake shaking," Earthquake Engineering \& Structural Dynamics, vol. 38, no. 6, pp. 827-848, 2009.

[11] A. Saraswat, G. R. Reddy, S. Ghosh, A. K. Ghosh, and A. Kumar, "Seismic stability of a standalone glove box 
structure," Nuclear Engineering and Design, vol. 276, pp. 178-190, 2014.

[12] A. Dar, D. Konstantinidis, and W. W. El-Dakhakhni, "Evaluation of ASCE 43-05 seismic design criteria for rocking objects in nuclear facilities," Journal of Structural Engineering, vol. 142, no. 11, Article ID 4016110, 2016.

[13] N. Makris and G. Kampas, "Size versus slenderness: two competing parameters in the seismic stability of free-standing rocking columns," Bulletin of the Seismological Society of America, vol. 106, no. 1, pp. 104-122, 2016.

[14] A. Gesualdo, A. Iannuzzo, M. Monaco, and F. Penta, "Rocking of a rigid block freestanding on a flat pedestal," Journal of Zhejiang University - Science, vol. 19, no. 5, pp. 331-345, 2018.

[15] A. A. Costa, A. Arêde, A. Penna, and A. Costa, "Free rocking response of a regular stone masonry wall with equivalent block approach: experimental and analytical evaluation," Earthquake Engineering \& Structural Dynamics, vol. 42, no. 15, pp. 2297-2319, 2013.

[16] A. Di Egidio, A. Contento, C. Olivieri, and A. M. de Leo, "Protection from overturning of rigid block-like objects with linear quadratic regulator active control," Structural Control and Health Monitoring, vol. 27, 2020.

[17] S. Sorace and G. Terenzi, "Seismic performance assessment and base-isolated floor protection of statues exhibited in museum halls," Bulletin of Earthquake Engineering, vol. 13, no. 6, pp. 1873-1892, 2015.

[18] A. Di Egidio, A. Contento, A. M. de Leo, and P. Gardoni, "Dynamic and seismic protection of rigid-block-like elements and structures on deformable ground with mass-damper dynamic absorbers," Journal of Engineering Mechanics, vol. 146, no. 6, Article ID 4020046, 2020.

[19] E. Cosenza, L. Di Sarno, G. Maddaloni, G. Magliulo, C. Petrone, and A. Prota, "Shake table tests for the seismic fragility evaluation of hospital rooms," Earthquake Engineering \& Structural Dynamics, vol. 44, no. 1, pp. 23-40, 2015.

[20] Y. Bao and D. Konstantinidis, "Dynamics of a sliding-rocking block considering impact with an adjacent wall," Earthquake Engineering \& Structural Dynamics, vol. 49, no. 5, pp. 498-523, 2020.

[21] A. Gesualdo, A. Iannuzzo, V. Minutolo, and M. Monaco, "Rocking of freestanding objects: theoretical and experimental comparisons," Journal of Theoretical and Applied Mechanics, vol. 56, pp. 977-991, 2018.

[22] A. Filiatrault, S. Kuan, and R. Tremblay, "Shake table testing of bookcase - partition wall systems," Canadian Journal of Civil Engineering, vol. 31, no. 4, pp. 664-676, 2004.

[23] M. F. Vassiliou, M. Broccardo, C. Cengiz et al., "Shake table testing of a rocking podium: results of a blind prediction contest," Earthquake Engineering \& Structural Dynamics, vol. 50, no. 4, pp. 1043-1062, 2021.

[24] C. E. Wittich and T. C. Hutchinson, "Shake table tests of stiff, unattached, asymmetric structures," Earthquake Engineering \& Structural Dynamics, vol. 44, no. 14, pp. 2425-2443, 2015.

[25] C. E. Wittich and T. C. Hutchinson, "Rocking bodies with arbitrary interface defects: analytical development and experimental verification," Earthquake Engineering \& Structural Dynamics, vol. 47, no. 1, pp. 69-85, 2018.

[26] M. F. Vassiliou, K. R. Mackie, and B. Stojadinović, "Dynamic response analysis of solitary flexible rocking bodies: modeling and behavior under pulse-like ground excitation," Earthquake Engineering \& Structural Dynamics, vol. 43, no. 10, pp. 1463-1481, 2014.

[27] C. Petrone, L. Di Sarno, G. Magliulo, and E. Cosenza, "Numerical modelling and fragility assessment of typical freestanding building contents," Bulletin of Earthquake Engineering, vol. 15, no. 4, pp. 1609-1633, 2017.

[28] M. Fragiadakis and S. Diamantopoulos, "Fragility and risk assessment of freestanding building contents," Earthquake Engineering \& Structural Dynamics, vol. 49, no. 10, pp. 1028-1048, 2020.

[29] D. D’Angela, G. Magliulo, and E. Cosenza, "Seismic damage assessment of unanchored nonstructural components taking into account the building response," Structural Safety, vol. 93, Article ID 102126, 2021.

[30] B. Huang, Q. Pan, W. Lu, and F. Shen, "Free-rocking tests of a freestanding object with variation of center of gravity," Earthquake Engineering \& Structural Dynamics, vol. 50, no. 11, pp. 3015-3040, 2021.

[31] L. Berto, T. Favaretto, A. Saetta, F. Antonelli, and L. Lazzarini, "Assessment of seismic vulnerability of art objects: the "Galleria dei Prigioni" sculptures at the Accademia Gallery in Florence," Journal of Cultural Heritage, vol. 13, no. 1, pp. 7-21, 2012.

[32] T. Neurohr and G. McClure, "Shake table testing of museum display cases," Canadian Journal of Civil Engineering, vol. 35, no. 12, pp. 1353-1364, 2008.

[33] C. Burningham, G. Mosqueda, and R. R. Saavedra, "Comparison of seismic fragility of free standing equipment using current testing protocols and recorded building floor motions," in Proceedings of the, Earthquake Engineering Symposium for Young Researchers, Seattle, Washington, DC, USA, 2003.

[34] Federal Emergency Management Agency (Fema), "Interim Protocols for Determining Seismic Performance Characteristics of Structural and Nonstructural Components through Laboratory testing," Report No. FEMA 461, Federal Emergency Management Agency (Fema), Washington, DC, USA, 2007.

[35] International Code Council Evaluation Service (Icc-Es), AC156 Acceptance Criteria for the Seismic Qualification of Nonstructural Components, International Code Council Evaluation Service (Icc-Es), Brea, CA, USA, 2012.

[36] G. Magliulo, G. Maddaloni, and C. Petrone, "A procedure to select time-histories for shaking table tests on nonstructural components," in Proceedings of the 4th ECCOMAS Thematic Conference on Computational Methods in Structural Dynamics and Earthquake Engineering, Kos Island, Greece, June 2013.

[37] C. Petrone, G. Magliulo, and G. Manfredi, "Shake table tests on standard and innovative temporary partition walls," Earthquake Engineering \& Structural Dynamics, vol. 46, no. 10, pp. 1599-1624, 2017.

[38] G. Magliulo, V. Pentangelo, G. Maddaloni et al., "Shake table tests for seismic assessment of suspended continuous ceilings," Bulletin of Earthquake Engineering, vol. 10, no. 6, pp. 1819-1832, 2012.

[39] C. Petrone, G. Magliulo, and G. Manfredi, "Shake table tests for the seismic assessment of hollow brick internal partitions," Engineering Structures, vol. 72, pp. 203-214, 2014.

[40] J. F. Chai and F. R. Lin, "Seismic retrofit and shaking table test of medical equipment in a hospital," in Proceedings of the Ninth Pacific Conference on Earthquake Engineering Building an Earthquake-Resilient Society, Auckland, New Zealand, January 2011.

[41] B. Huang, S. Günay, and W. Lu, "Seismic assessment of freestanding ceramic vase with shaking table testing and performance-based earthquake engineering," Journal of Earthquake Engineering, pp. 1-23, 2021, https://www. tandfonline.com/doi/full/10.1080/13632469.2021.1979132?scr oll=top\&needAccess $=$ true. 
[42] T. Mahdi and A. Mahdi, "Sliding fragility of restrained and unrestrained block-type nonstructural components," Asian Journal of Civil Engineering, vol. 14, pp. 545-555, 2013.

[43] American Society of Civil Engineers, Ed., ASCE 7-16. Minimum Design Loads for Buildings and Other Structures, American Society of Civil Engineers : Structural Engineering Institute, Reston, VA, USA, 2017.

[44] L. Di Sarno, C. Petrone, G. Magliulo, and G. Manfredi, "Dynamic properties of typical consultation room medical components," Engineering Structures, vol. 100, pp. 442-454, 2015.

[45] J. M. Bracci, A. M. Reinhorn, and J. B. Mander, "Seismic Resistance of Reinforced concrete Frame Structures Designed Only for Gravity Loads: Part I - Design and Properties of a One-Third Scale Model Structure," Technical Report NCEER92-0027, State University of New York at Buffalo, New York, NY, USA, 1992.

[46] R. W. Clough and J. Penzien, Dynamics of Structures, McGraw-Hill, New York, NY, USA, 1975.

[47] A. K. Chopra, Dynamics of Structures: Theory and Applications to Earthquake Engineering, Pearson/Prentice Hall, Hoboken, New Jersey, . edition, 2007.

[48] G. A. Papagiannopoulos and G. D. Hatzigeorgiou, "On the use of the half-power bandwidth method to estimate damping in building structures," Soil Dynamics and Earthquake Engineering, vol. 31, no. 7, pp. 1075-1079, 2011.

[49] Federal Emergency Management Agency (Fema), "Reducing the Risks of Nonstructural Earthquake Damage - A Practical Guide," Report No. FEMA E-74, Federal Emergency Management Agency (Fema), Washington, DC, USA, 2012. 